\title{
Amyloid precursor protein localises to ependymal cilia in vertebrates and is required for ciliogenesis and brain development in zebrafish
}

\section{Jasmine Chebli}

Institute of Neuroscience and Physiology, Department of Psychiatry and Neurochemistry, The Sahlgrenska Academy, University of Gothenburg

\section{Maryam Rahmati}

Institute of Neuroscience and Physiology, Department of Psychiatry and Neurochemistry, The

Sahlgrenska Academy, University of Gothenburg

\section{Tammaryn Lashley}

Department of Neurodegenerative Disease, UCL Institute of Neurology, Queen Square, London

\section{Brigitta Edeman}

Department of Laboratory Medicine, University of Gothenburg

\section{Anders Oldfors}

Department of Laboratory Medicine, University of Gothenburg

\section{Henrik Zetterberg}

Clinical Neurochemistry Laboratory, Sahlgrenska University Hospital, Mölndal

Alexandra Abramsson ( $\nabla$ alexandra.abramsson@neuro.gu.se)

Institute of Neuroscience and Physiology, Department of Psychiatry and Neurochemistry, The Sahlgrenska Academy, University of Gothenburg

\section{Research Article}

Keywords: Amyloid precursor protein (APP), orthologues, Appa and Appb, APLP1, APLP2, amyloid-beta peptide $(A \beta)$

Posted Date: July 8th, 2021

DOI: https://doi.org/10.21203/rs.3.rs-629681/v1

License: (c) (1) This work is licensed under a Creative Commons Attribution 4.0 International License. Read Full License 


\section{Amyloid precursor protein localises to ependymal cilia in vertebrates and is required for ciliogenesis and brain development in zebrafish}

Jasmine Chebli ${ }^{1}$, Maryam Rahmati ${ }^{1}$, Tammaryn Lashley ${ }^{2,3}$, Birgitta Edeman ${ }^{4}$, Anders Oldfors ${ }^{4}$, Henrik Zetterberg ${ }^{1,3,5,6}$ and Alexandra Abramsson ${ }^{1 *}$

${ }^{I}$ Institute of Neuroscience and Physiology, Department of Psychiatry and Neurochemistry, The Sahlgrenska Academy, University of Gothenburg, Gothenburg, Sweden.

${ }^{2}$ Queen Square Brain Bank for Neurological Disorders, Department of Clinical and Movement 11 Neurosciences, Queen Square Institute of Neurology, University College London, London, UK.

${ }^{3}$ Department of Neurodegenerative Disease, UCL Institute of Neurology, Queen Square, London, United Kingdom.

${ }^{4}$ Department of Laboratory Medicine, University of Gothenburg, Gothenburg, Sweden.

${ }^{5}$ Clinical Neurochemistry Laboratory, Sahlgrenska University Hospital, Mölndal, Sweden.

*Corresponding author: alexandra.abramsson@neuro.gu.se. Institute of Neuroscience and Physiology, Department of Psychiatry and Neurochemistry, The Sahlgrenska Academy, University of Gothenburg, S-41345 Gothenburg, Sweden.

\section{Abstract}

Amyloid precursor protein (APP) is ubiquitously expressed in human, mice and in zebrafish. In zebrafish, there are two orthologues, Appa and Appb. Interestingly, some cellular processes associated with APP overlap with cilia-mediated functions. Whereas the localization of APP to primary cilia of in vitro-cultured cells has been reported, we addressed the presence of APP in motile and in non-motile sensory cilia and its potential implication for ciliogenesis using zebrafish, mouse, and human samples. We report that Appa and Appb are expressed by ciliated cells and become localized at the membrane of cilia in the olfactory epithelium, otic vesicle and 
in the brain ventricles of zebrafish embryos. App in ependymal cilia persisted in adult zebrafish and was also detected in mouse and human brain. Finally, we found morphologically abnormal ependymal cilia and smaller brain ventricles in $a p p a^{-/-} a p p b^{-/-}$mutant zebrafish. Our findings demonstrate an evolutionary conserved localisation of APP to cilia and suggest a role of App in ciliogenesis and cilia-related functions.

\section{Introduction}

Amyloid precursor protein (APP) is a ubiquitously expressed type-1 transmembrane protein that, together with the APP-like protein 1 and -2 (APLP1, APLP2), comprises the APP gene family. In addition to their various splice forms, they are all post-translationally modified through proteolytic processing ${ }^{1}$. Although the physiological relevance of the fragments generated is not fully understood, one of these, the amyloid-beta peptide $(\mathrm{A} \beta)$ originating from the transmembrane domain of the APP protein, is the main component of brain amyloid plaques in Alzheimer's disease (AD) ${ }^{1,2}$. Beyond its pathological involvement, studies on APP have revealed essential physiological functions including neurogenesis ${ }^{3,4}$, neurite outgrowth ${ }^{5,6}$, adhesion properties ${ }^{6,7}$, synapse formation ${ }^{8}$, and neuronal migration ${ }^{6,9,10}$. Nevertheless, the involvement of each APP family member in these processes remains unclear, since redundancy makes it difficult to unravel the contribution of a specific protein ${ }^{11}$. Although the molecular mechanisms behind the APP-related processes are yet to be determined, accumulating evidence support that APP orchestrates cellular processes through receptor-like interactions with both inter- and intra-cellular signaling molecules ${ }^{6}$.

51 The cilium is a highly conserved organelle across species, which contributes to a wide range of 52 cellular processes ${ }^{12}$. Cilia can broadly be categorized into motile and non-motile. Non-motile cilia include primary cilia, which are ubiquitously expressed on most cells as a single short 
antenna-like structure, and sensory cilia, that are only expressed by specific cells. Primary cilia are enriched in receptors and sites for inter-cell signaling transduction and are notably implicated in cell division, autophagy, midbrain development, memory and learning abilities ${ }^{13}$. As for the sensory cilia, they are notably found in the otic vesicle as stereocilia and kinocilia. Motile cilia are present on cells involved in fluid movement including the epithelium of the respiratory tract and the ependymal layer of the brain ventricles. Ependymal cells are derived from radial glial cells and when fully differentiated are decorated with tufts of motile cilia anchored with roots at the apical cellular membrane ${ }^{12,14}$. The coordinated periodic beating of the cilia participate in the generation of cerebrospinal fluid (CSF) flow within ventricle cavities 15. Circulation of CSF is believed to facilitate transfer of signaling molecules and removal of metabolic waste products to prevent accumulation of neurotoxic residues in the brain parenchyma ${ }^{16-18}$.

There are several findings supporting a connection between APP and cilia. First, part of the wide range of cilia-mediated functions overlap with processes linked to APP, e.g., cognitive impairment ${ }^{19}$, differentiation of neurons ${ }^{20}$, formation of corpus callosum ${ }^{19,21}$, neuronal migration ${ }^{22-24}$ and sensing of guidance molecules ${ }^{25}$. Second, overexpression of APP impairs primary cilia both in a mouse AD model and in individuals with Down syndrome, harboring three copies of APP ${ }^{26,27}$. The latter is also associated with decreased CSF flow and accumulation of CSF (hydrocephalus), two phenotypes commonly associated with defects in motile cilia ${ }^{28}$. Finally, APP has been shown to localize to primary cilia in vitro and A $\beta$ exposure results in reduced cilia length ${ }^{29}$. Taken together, these findings warrant further investigations of the role of APP in both motile and non-motile cilia.

In the present study, we address the presence of APP in motile and non-motile (sensory) cilia and its possible functions using zebrafish, mouse and human samples. We found that the zebrafish App homologues are expressed by ciliated cells and become localized at the 
membrane of cilia in the otic vesicle, the nasal epithelium, and the brain ventricles of zebrafish embryos. The presence of App in ependymal cilia persisted in adult zebrafish and was also detected in the ependymal cells of mouse and human brains. In addition, we show that zebrafish embryos with mutations in both app paralogues $\left(a p p a^{-/} a p p b^{-/}\right)$have morphologically abnormal ependymal cilia and smaller brain ventricles compared with wild-type siblings.

\section{Results}

\section{Appa and appb mRNA expression patterns at the brain ventricular limits}

The zebrafish app genes, appa and appb, are expressed in the CNS, and have both distinct and shared expression patterns ${ }^{7,30}$. Due to the lack of specific antibodies, we used fluorescent whole mount in situ hybridization to increase the cellular resolution of appa and appb mRNA expression in areas with motile cilia on $30 \mathrm{hpf}$ wild-type larvae zebrafish (Figure 1). Consistent with previous studies, we observed appa mRNA expression in the lens, the olfactory bulb and epithelium, in the trigeminal ganglia and in the otic vesicle. (Figure $1 C$ ). Similarly, the appb mRNA expression signal corroborated previous data on appb mRNA expression ${ }^{30}$ in the olfactory and otic vesicle epithelia (Figure 1H).

In addition, both appa (Figures $1 C$-G and high magnification Figures $1 N, O$ ) and appb (Figures $1 \boldsymbol{H}-\boldsymbol{L}, \boldsymbol{P}, \boldsymbol{Q}$ ) mRNA signals labelled cells lining the diencephalic ventricle both in the dorsal and ventral areas. Negative controls did not show any specific signal (Supplementary file 1). Together, these results show expression of appa and appb in areas with ciliated cells, including cells lining brain ventricles, otic vesicle and olfactory organ, thus suggesting a possible role of App in cilia formation and function. 
Figure 1. Expression of appa and appb mRNA in zebrafish larvae

A

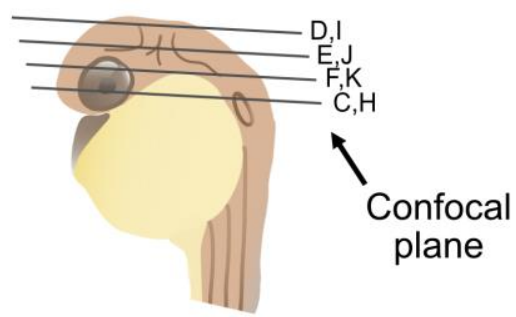

$\mathrm{B}$

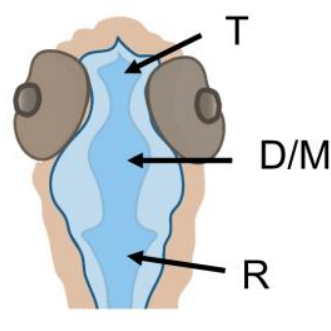

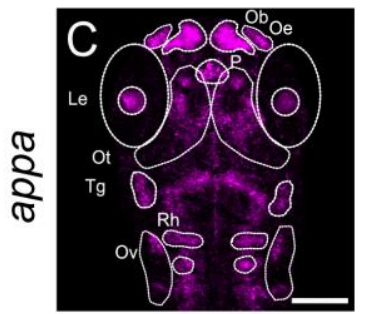

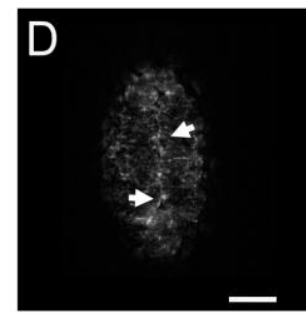

E

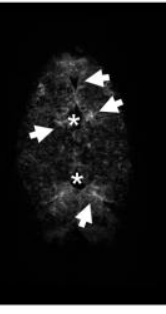

F
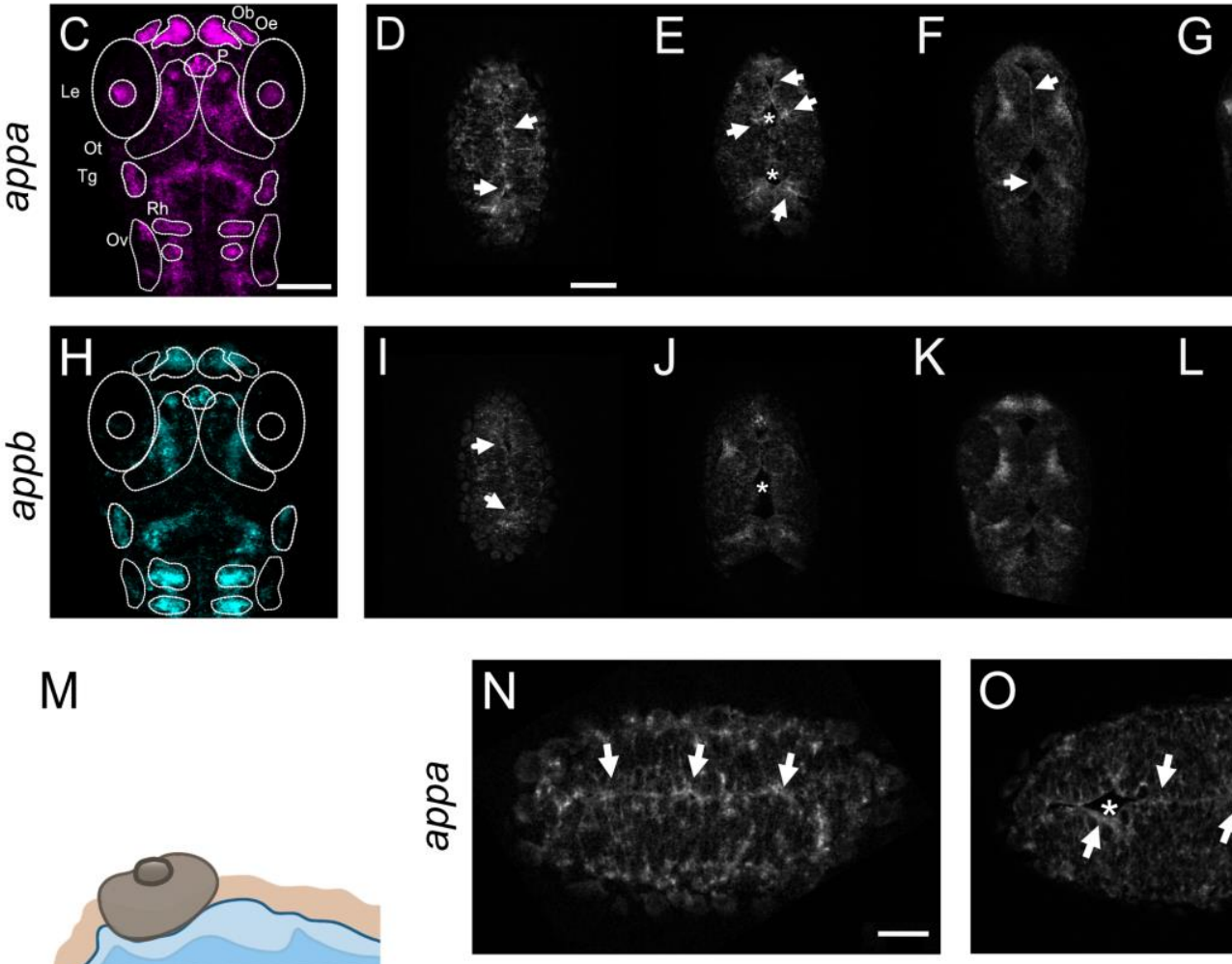

M
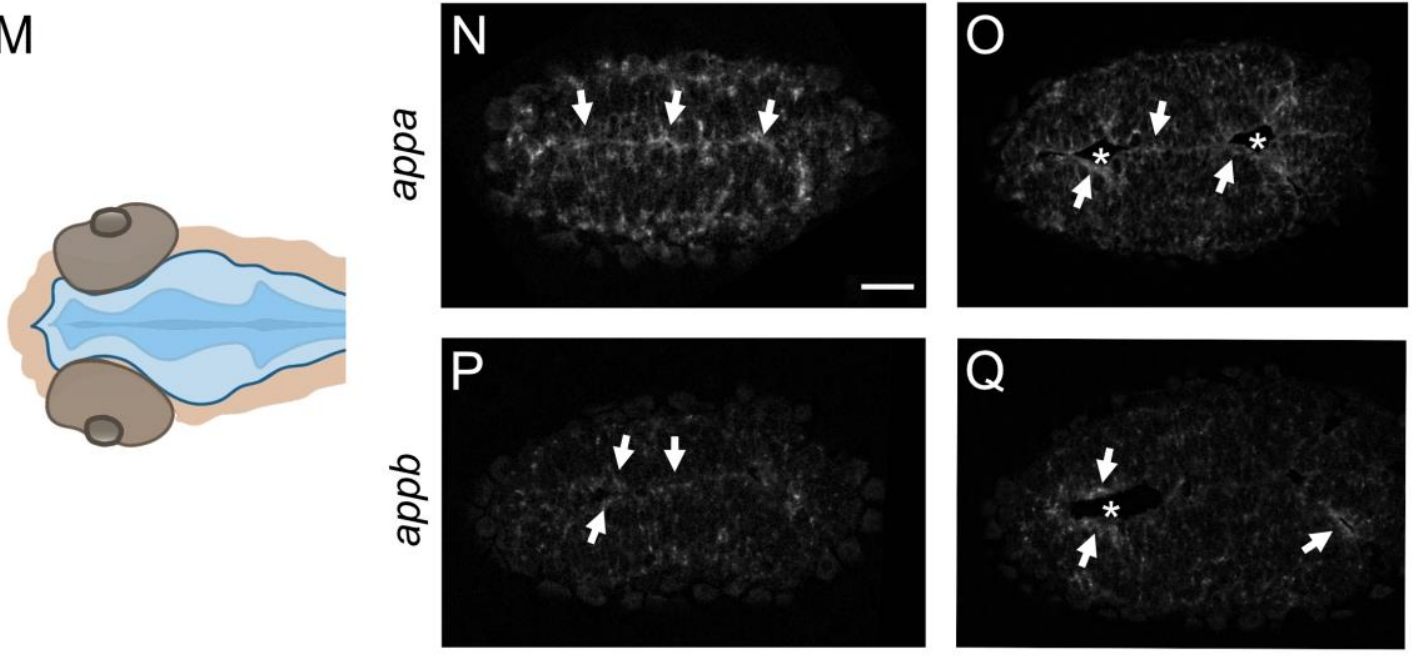
107 The expression of both appa and appb in ciliated cells made us ask if the proteins become

108 distributed out to the cilia. The zebrafish olfactory epithelium and the otic vesicle comprise

109 ciliated cells and are regions where both appa and appb mRNAs are expressed. To address if 110 Appa and Appb become localized to these cilia, we performed immunofluorescent staining on 111 zebrafish larvae.

\section{Olfactory sensory neuron cilia}

113 We used the Y188 antibody, binding to a conserved epitope in the C-terminal end of human, 114 mouse, and zebrafish App (Figure 6C), in combination with the anti-acetylated tubulin 115 antibody, labelling microtubule structures of cilia. Immunofluorescent co-labelling detected a 116 punctate App signal in the heavily ciliated olfactory epithelium area at 30 hpf (Figure 2A). 117 However, while the resolution of the images did not allow distinction between each cilium, App 118 signal seemed to localize to most of them. In addition to the cilium, App expression was also 119 found at the base of these motile cilia (Figure $2 \boldsymbol{A}^{\prime}$ ').

Otic vesicle cilia

121 Similar to the olfactory neurons, high accumulation of App was noted at the base of the cilia in

122 the otic vesicle. In zebrafish, hair cells of the otic vesicle have two types of cilia, a long single 123 kinocilium and a bundle of shorter stereocilia ${ }^{31}$. The immunofluorescent staining revealed App 124 expression in both types of cilia at early time points in the larvae development (Figures $2 B-C$ ). 125 Staining of $24 \mathrm{hpf}$ larvae with glutamylated tubulin, highlighting the cilia basal body, clearly 126 showed an App signal within the hair cells and close to the basal body (Figures $2 \boldsymbol{B}, \boldsymbol{B}$ ', $\boldsymbol{B}$ ') ).

127 App expression became more distinct at $30 \mathrm{hpf}$ (Figure $2 C$ ). Plots of the intensity profile of 128 App (green) and acetylated tubulin (magenta) showed a punctate distribution of App throughout 
129 the kinocilium (Figure 2D), which supports that App localizes to the cilium membrane (Figure

$130 \boldsymbol{2 E}$ ). No signal was detected in the intensity profile in the absence of App puncta (Figure $2 \boldsymbol{F}$ ),

131 and the negative control (absence of primary antibody) was negative (Supplementary file 2).

132 Together, these data show expression of App in cilia and ciliated cells of the otic vesicle and

133 olfactory bulb and indicate that App is located at the cilia membrane.

Figure 2. App protein is localized to non-primary cilia in zebrafish larvae
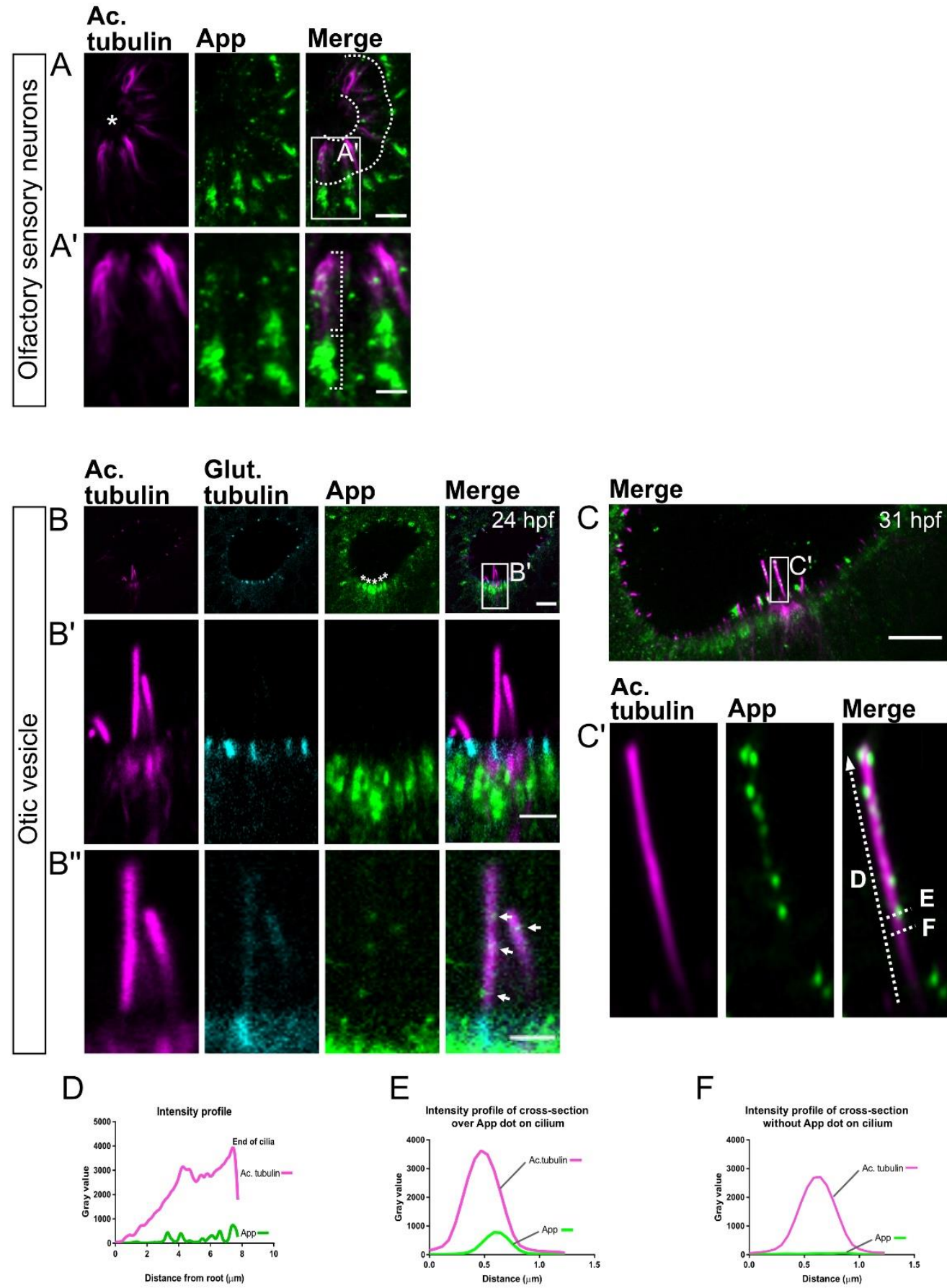
137 As APP was previously shown to be expressed by the ependymal cells in rodents and in humans

$138 \quad 32-34$, we explored App expression by ependymal cells and App localization at their cilia in

139 larvae and adult zebrafish (Figure 3). At $30 \mathrm{hpf}$, the brain ventricles are inflated and the

140 differentiation of motile cilia in the most ventral and dorsal regions have just started but do not

141 yet contribute to directional CSF flow ${ }^{35}$. This facilitates whole-mount imaging and

142 measurement of single cilium. Using the same combination of antibodies (anti-APP (Y188) and

143 anti-acetylated tubulin) as above, we could detect App-positive puncta along the acetylated

144 tubulin signal in most cilia (Figures 3B,C). To address if App localization to cilia is maintained

145 into adulthood, we performed immunofluorescent staining on coronal sections of adult

146 zebrafish brains using antibodies detecting App (Y188) and acetylated tubulin to label cilia.

147 Our results showed that consistent to larvae, App was distributed to ependymal cilia in the adult

148 brain. In contrast to larvae, ependymal cells in adult individuals were covered with multiple

149 motile cilia. Cryosections of adult zebrafish brain revealed dense cilia tufts with App-positive

150 staining at the apical side of the ependymal cells (Figures $3 \boldsymbol{E}, \boldsymbol{F}$ ). Furthermore, App was also

151 expressed by ependymal cells, similarly to what has previously been described in rodents and

152 humans (Figure $3 \boldsymbol{F}$ ). Negative controls did not show any cilia-specific staining

153 (Supplementary file 3). 
Figure 3. App is localized to the cilia membrane of neuroepithelium and ependyme in larvae and adult zebrafish

Zebrafish larvae

A

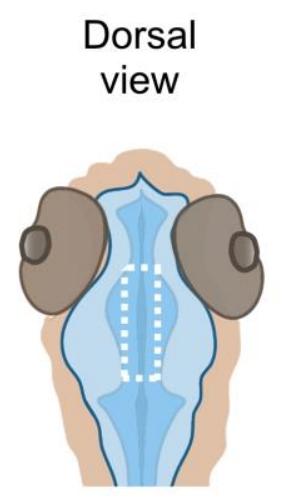

Zebrafish adult

D

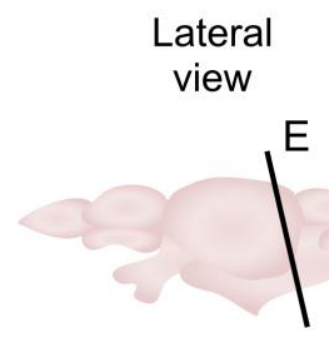

F Ac. tubulin

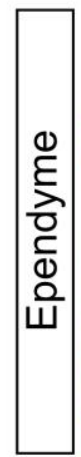

B

Merge
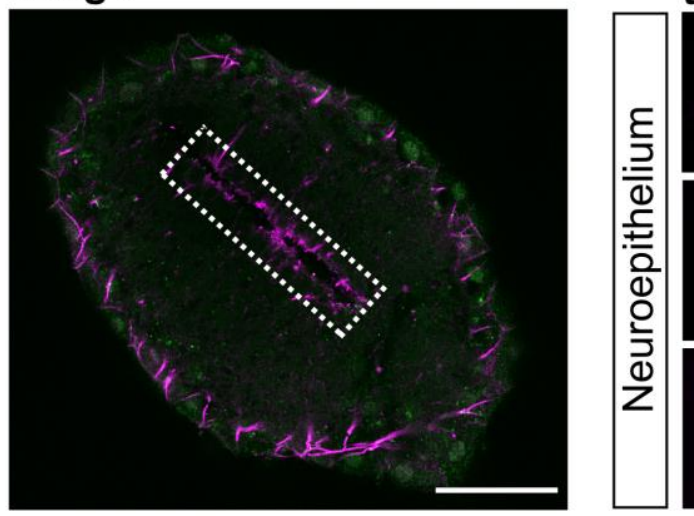

Ac. tubulin App Merge

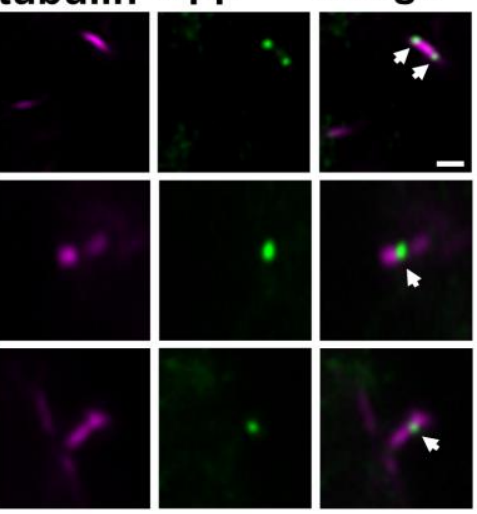

App

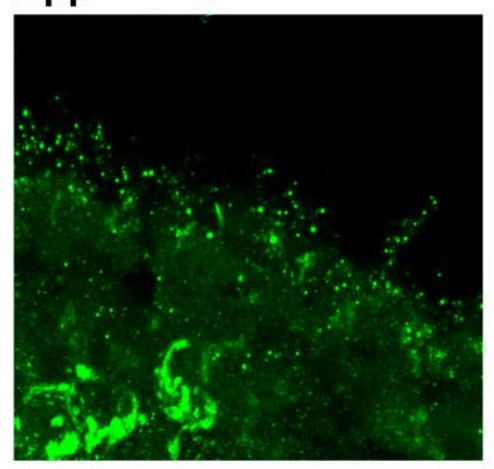

E

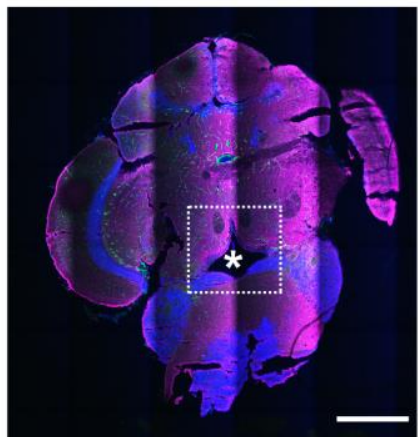

Merge

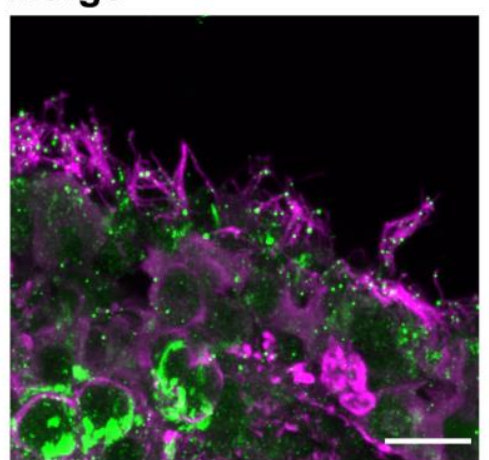


159 APP is also localized to ependymal cilia in mice and humans. We performed immunostaining

160 on mouse brain sections using two antibodies directed to APP, Y188 binding to the C-terminal

161 intra-cellular domain and 22C11 detecting the E1 domain of the N-terminal region (Figure 6C),

162 together with anti-acetylated tubulin. The ependymal motile cilia were easily localized in the

163 third ventricle of the brain sagittal section (Figures $\mathbf{4 A , B}$ ). Congruent with our results on adult

164 zebrafish brains, we detected strong APP expression with both antibodies throughout the 165 ependymal cells layer and punctate APP staining (Y188 see Figure 4C and 22C11 see Figure

$1664 D$ ) overlapping with acetylated tubulin-positive cilia. Interestingly, APP expression by the

167 choroid plexus cells was detectable (Figure $4 \boldsymbol{B}$ ). Negative control for primary antibodies was

168 performed and showed no or weak signal (Supplementary file 4). 
Figure 4. APP is localized to the ependymal cilia in adult mouse
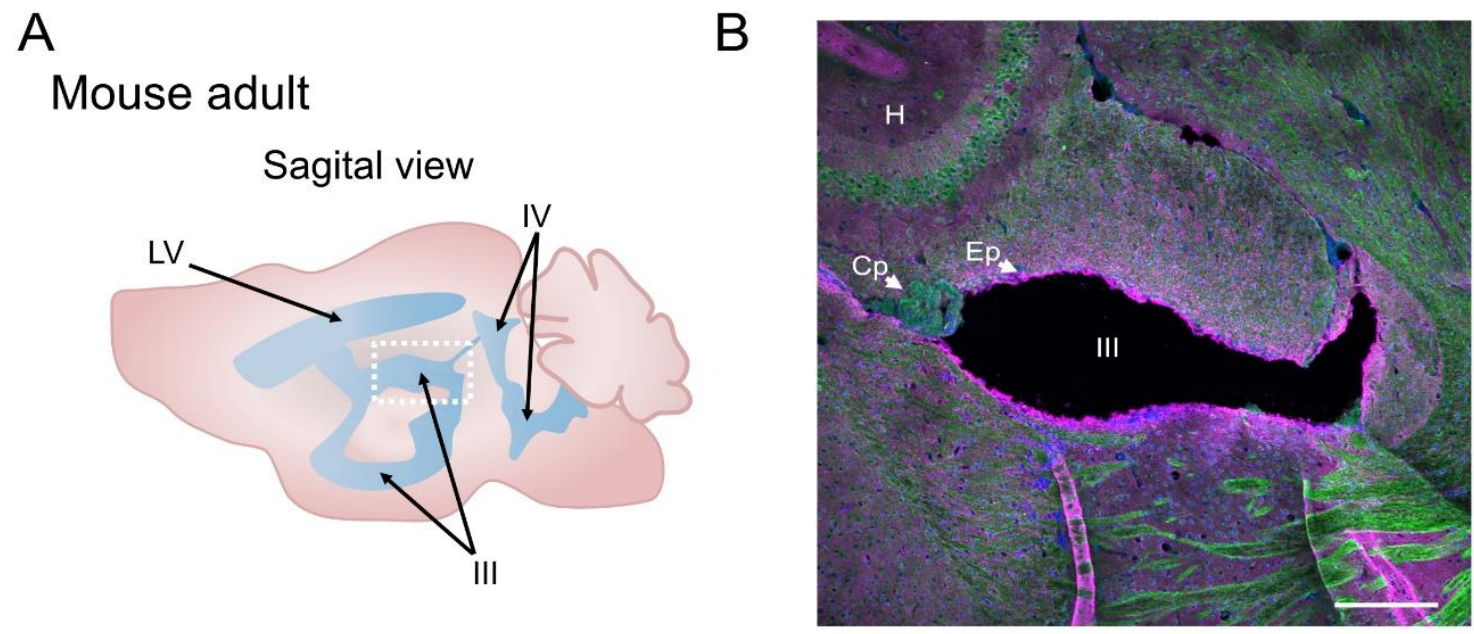

Ependyme

C

\section{DAPI}

Ac. tubulin

APP (Y188)
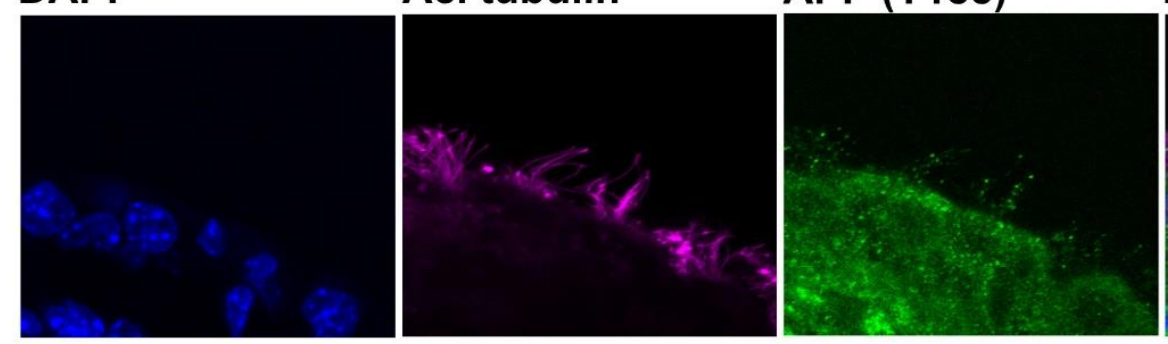

Merge

D

\section{DAPI}

Ac. tubulin

APP (22C11)

\section{Merge}
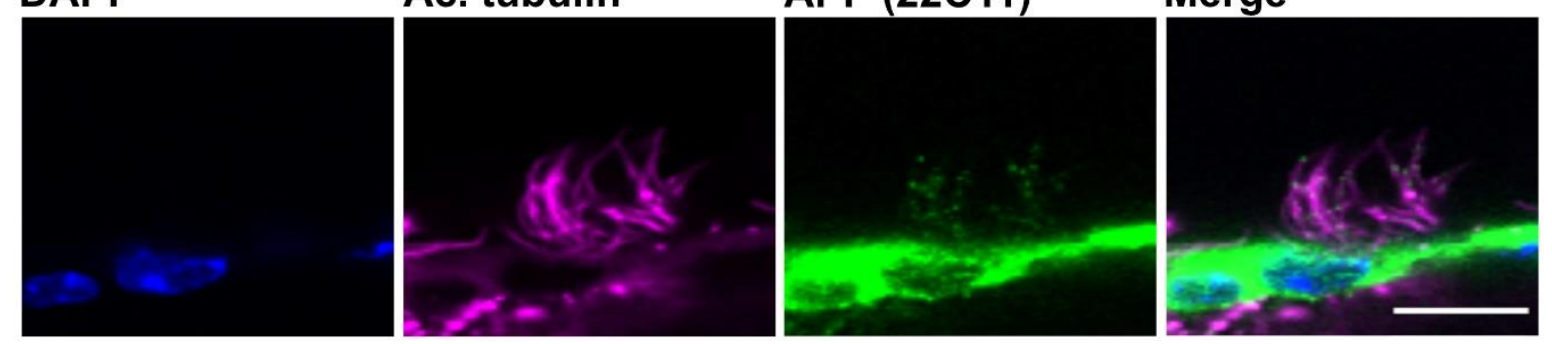

171

172 
173 In the human brain, acetylated tubulin staining allowed separation of cellular layers of the

174 caudate nucleus and identification of acetylated tubulin-positive cilia of the ependymal cell

175 layer lining the lateral ventricle (Figures $\mathbf{5 A , B , D}$ ). However, while many ependymal cells had

176 intact cilia, many were found broken and dislocated from their cell (Supplementary file 5).

177 To address the presence of APP in ependymal cilia, brain serial sections of the caudate nucleus

178 were incubated with horseradish peroxidase (HRP)-conjugated Y188 or A8717 antibodies, both

179 recognizing the C-terminal domain of APP. Similarly to our results obtained in mouse and

180 zebrafish brains, brightfield images confirmed strong APP expression in the ependymal cells

181 and, upon higher magnification, in ependymal cilia (Figures 5C,E, Supplementary file 5). In

182 contrast to zebrafish and mouse, APP in human ependymal cilia was evenly distributed and was

183 not detected as puncta.

184 In summary, these results show that the expression of APP in the ependymal cells and their cilia

185 are conserved between species as far apart as zebrafish, mice, and humans. 
Figure 5. APP is localized to human brain ependymal cilia

\section{A}

\section{Ac. tubulin}

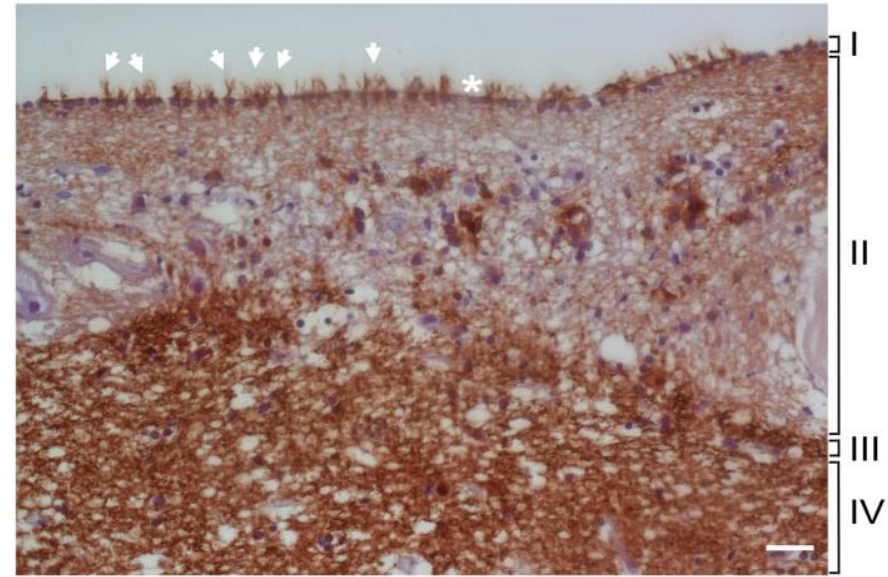

B

C

Ac. tubulin

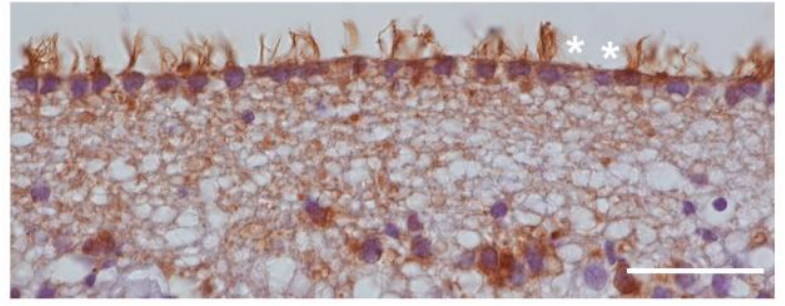

D

Ac. tubulin

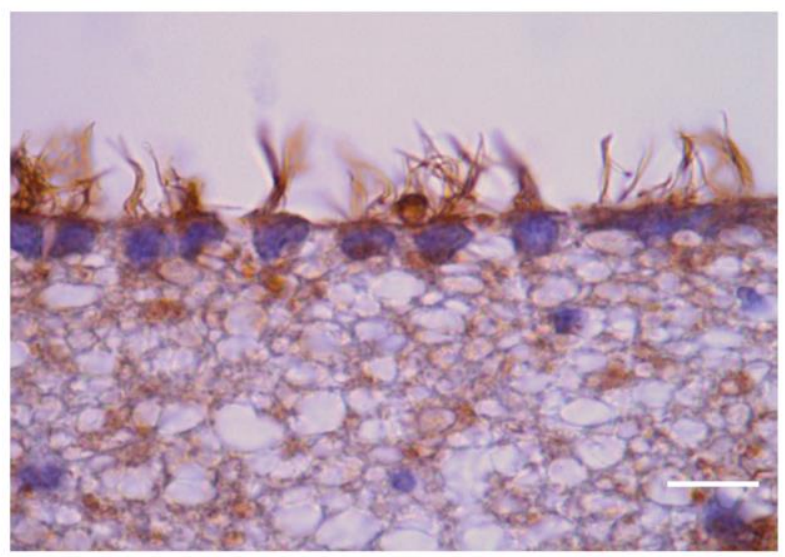

APP

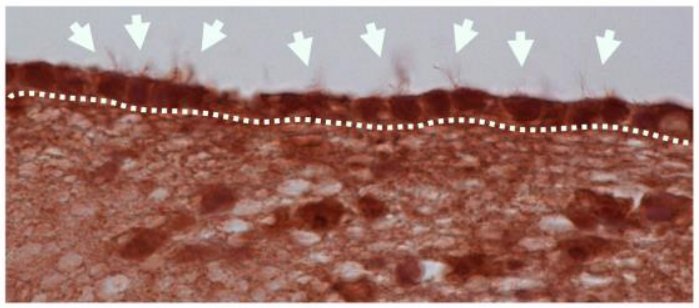

E

\section{APP}

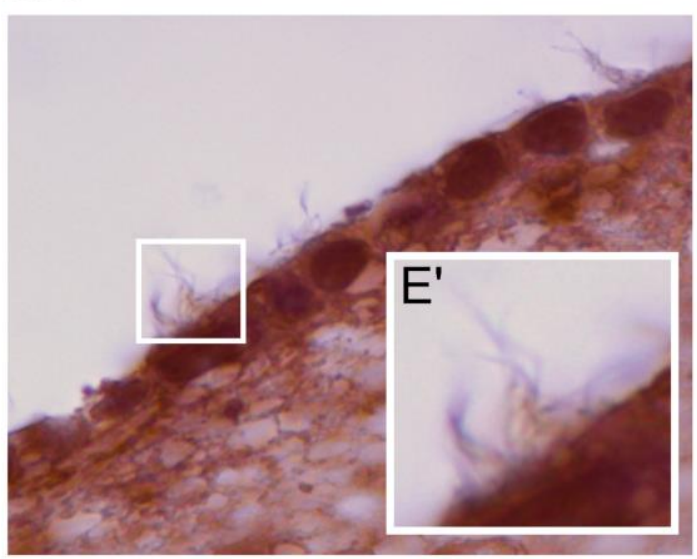


190 In contrast to humans and mice, zebrafish have two APP orthologues, appa and appb (together 191 designated $a p p$ ). Zebrafish with mutated $a p p b$ gene was generated and described by our lab 192 previously ${ }^{7}$. However, to investigate the requirement of both App proteins in ciliogenesis, we 193 used the CRISPR/Cas9 method to generate mutations in the zebrafish appa gene (Figure 6A).

194 A mutation was identified in exon 2 (Figure 6A), and Sanger sequencing confirmed a frame

195 shift mutation of 10 nucleotides (Figure $6 \boldsymbol{B}$ ). The mutation resulted in a premature stop codon 196 that is predicted to give rise to a protein truncation at amino acid 109 (Figure 6C). The appa 197 mutant allele was outcrossed into the $\mathrm{AB}$ background until generation $\mathrm{F} 4$ and then bred with 198 the $a p p b-/-$ to generate the double mutant $a p p a^{-/} a p p b^{-/-}$zebrafish line. The app mutant zebrafish 199 were healthy and fertile and did not show any gross morphological phenotypes. qPCR analysis 200 of genes expression showed very low appa and appb mRNA levels in the double mutant fish 201 line (Figure 6D). Western blot analysis using the Y188 and 22C11 antibodies with epitopes in 202 the intracellular and extracellular domain, respectively, showed decreased protein levels in app 203 double-mutant larvae (Figure 6E and Supplementary file 6). Both antibodies are likely cross204 reacting with Aplp2 since the epitope sequences are highly similar. These data show that the 205 introduced mutation in appa resulted in a significant decrease of both transcription and 206 translation of the Appa protein indicating that the mutation give rise to a loss-of-function 207 mutation. 
Figure 6. Generation of appa-- zebrafish

A

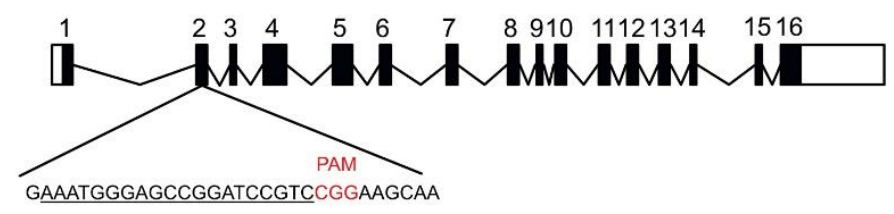

B

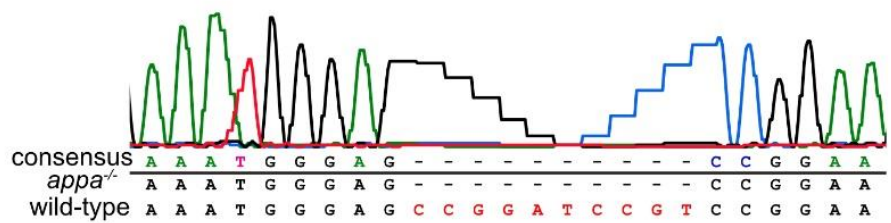

C

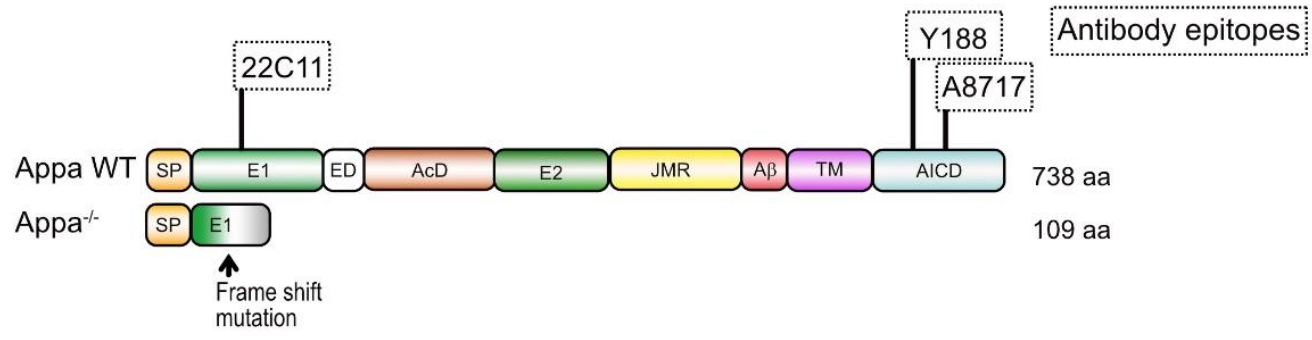

D
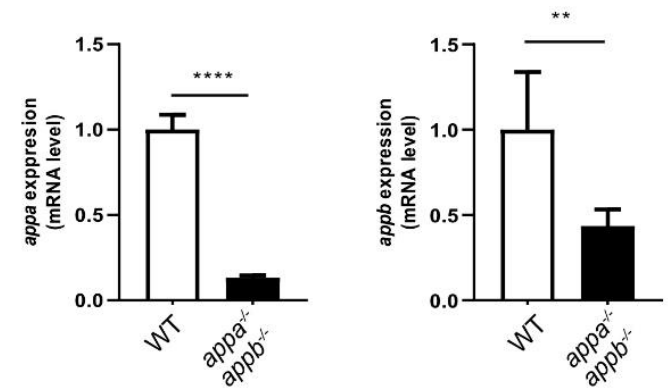

E

Western blot embryo 3dpf

22C11

Y188
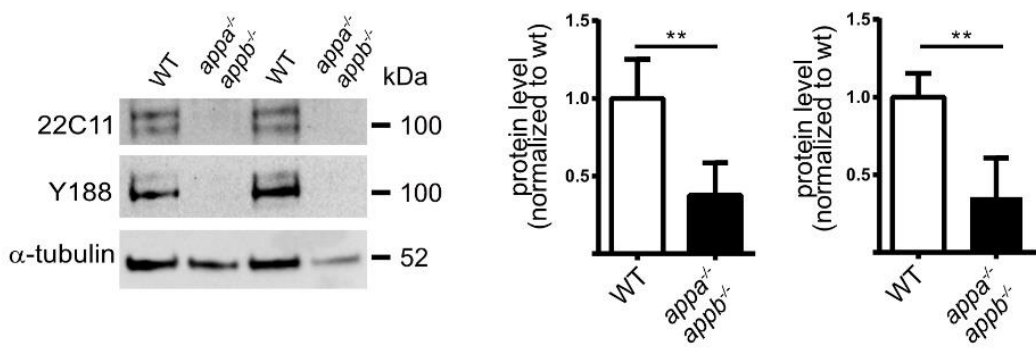
212 The conserved distribution of APP in brain ventricle cilia prompted us to address the 213 requirement of App during ciliogenesis. We measured the length of cilia in the midbrain 214 ventricle detected by acetylated tubulin immunostaining signal in both $a p p a^{-/} a_{p p b^{-/-}}$double 215 mutants and wildtype larvae at $30 \mathrm{hpf}$. At this stage, the cilia delineating the dorsal and ventral 216 parts of the diencephalic ventricles are not yet motile ${ }^{35}$. A 3D-region of interest (ROI) was used 217 to measure cilia length. The ROI was established from the dorsal part of the midbrain ventricle 218 to the ventricular space at a depth of around $25 \mu \mathrm{m}$. To our surprise, we found that the 219 ependymal cilia in the ROI were significantly longer in $a p p a^{-/-} a p p b^{-/-}$mutants compared with 220 wild-type larvae (Figure 7), which was confirmed by frequency distribution (Supplementary 221 file 7).

Figure 7. Longer cilia of brain ventricle neuroepithelium in appa--appb-/ larvae zebrafish

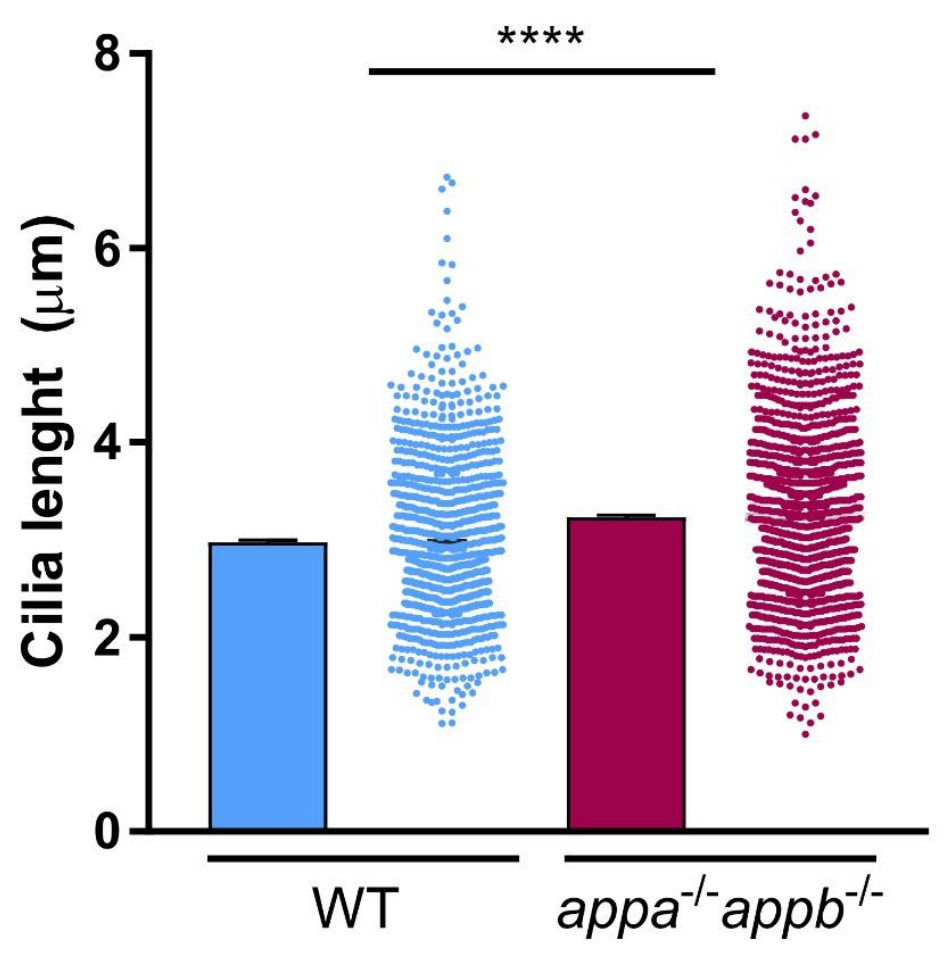




\section{cilia in appa $^{-/}$appb $^{-/-}$mutant adult zebrafish}

226 Emerging from the basal body is the axoneme, which forms the core of the cilium. First 227 described in the early 1950s with electron microscopy, axonemes are composed of nine 228 microtubule doublets at the periphery $(9+0)^{36}$. In some cilia, an additional central doublet is 229 expressed $(9+2)$, allowing cilia to generate and regulate movement ${ }^{37,38}$. This central 230 microtubule doublet is found in motile ependymal cilia $(9+2)$. To better characterize the ciliary 231 ultrastructure of App-deficient zebrafish, we performed transmission electron microscopy 232 (TEM) analysis of ependymal cells in adult zebrafish brains. TEM revealed a normal $(9+2)$ 233 axoneme in the cross-sections of ependymal cilia of WT (n=3) brain ventricle (Figures $8 \boldsymbol{A}-\boldsymbol{D})$.

234 In appa $^{-/} a_{p p b}{ }^{-/}$zebrafish $(\mathrm{n}=4)$, ependymal cilia showed normal $(9+2)$ axonemes (Figures $8 \boldsymbol{E}-$ $235 \boldsymbol{H})$.

Figure 8. Structural integrity of ependymal cilia in WT and appa-^apph ${ }^{-/}$zebrafish

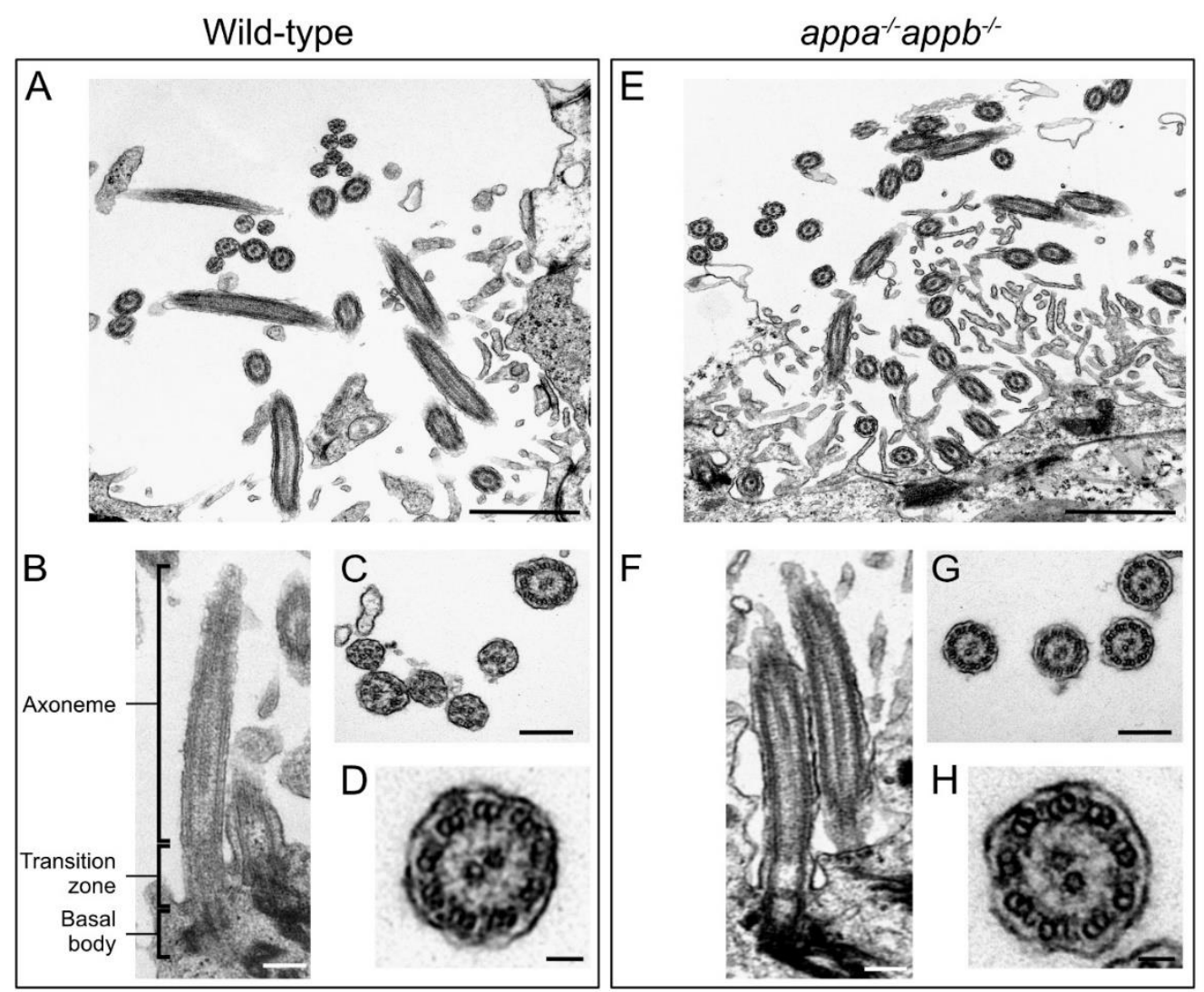


240 We then went on to address if defects in ependymal cilia affect brain ventricle formation. We 241 analysed brain ventricle volume and area in 2dpf larvae (Figure 9A) and found significant

242 reductions in both area and volume of the ventricular space in appa $^{-/} a_{p p b^{-/}}$compared with 243 wild-type (Figure 9B). These reductions were also observed when only the diencephalic 244 ventricle was analysed (Figure 9C) and compared between both genotypes (Figure 9D). The 245 gross morphology was next determined by measuring the length between specific points and 246 areas of the ventricles: rostral to caudal, diencephalon ventricle sagittal length, amplitude and 247 height (Figure 9E). However, no significant change was detected compared with wildtype 248 larvae (Figure 9F). These results show that while the overall brain morphology of App mutants 249 is maintained, their ventricles are smaller. 
Figure 9. appa--apph ${ }^{-/}$larvae zebrafish exhibit smaller brain ventricles

A

Dorsal view

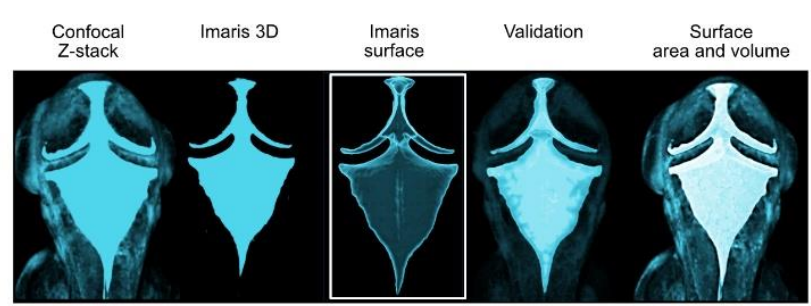

C
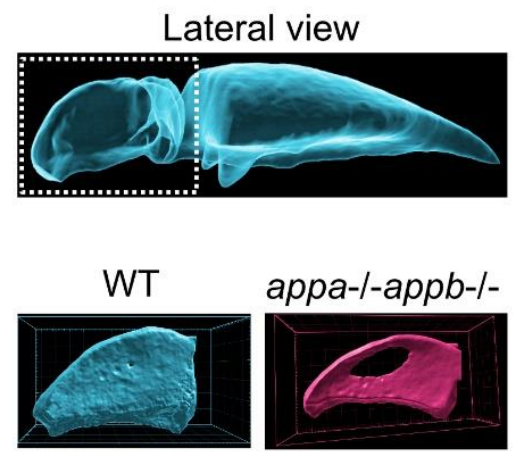

E

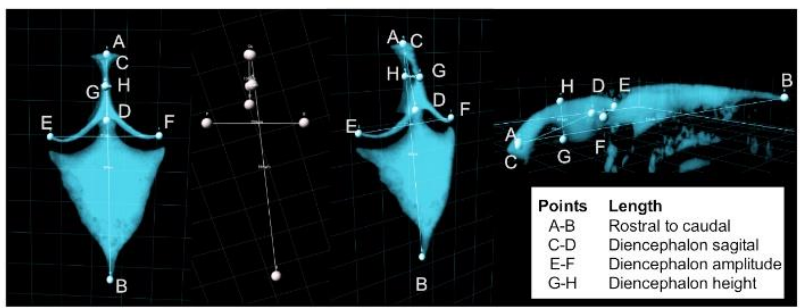

B

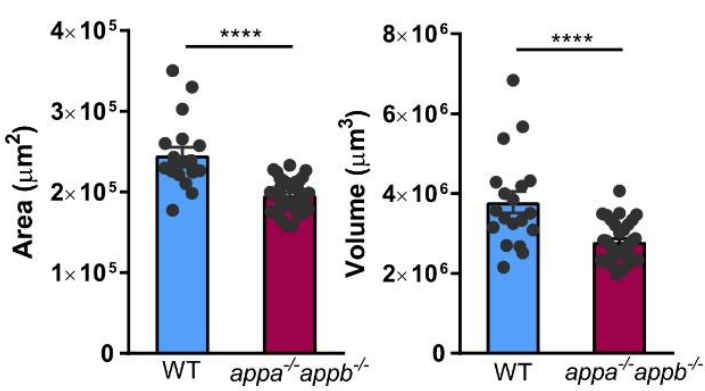

Diencephalic ventricle
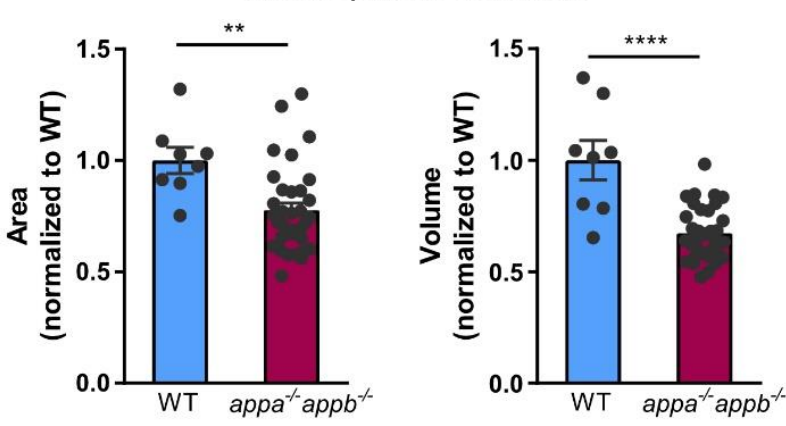

$\mathrm{F}$

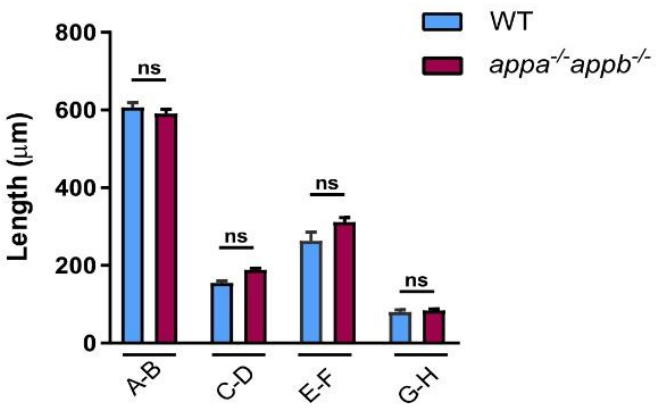


254 Many proteins distributed to the cilium carry one or more cilia targeting sequences (CTS). The 255 most common and well-studied are the $\mathrm{VxP}$ and AxxxQ motifs, both of which the requirement 256 has been shown in transmembrane proteins including opsins ${ }^{39-41}$ and somatostatin receptor 3 257 (SSTR3) ${ }^{42,43}$. The presence of App in cilia therefore made us investigate the presence of these 258 motifs in App. Interestingly, we found several different CTS motifs with most localized to the 259 mid- and C-terminal domain of the App protein (Supplementary file 8). Furthermore, most of these are in conserved regions and are thus shared between zebrafish, mouse and human (Supplementary file 8).

\section{Discussion}

265 In this study, we show that App localizes to several different non-motile and motile cilia in 266 zebrafish larvae including the stereo- and kinocilia of the otic vesicle, motile cilia of olfactory sensory neurons in the olfactory epithelium and cilia of the ependymal cells lining the brain ventricles. We also show an evolutionary conserved localization of APP to cilia of the ependymal cells lining the brain ventricles of adult zebrafish, mice and humans. As these results

270 indicated a possible function of APP in ciliogenesis or cilia function, we used zebrafish lacking 271 the two APP orthologues, Appa and Appb, and found longer ependymal cilia and smaller brain 272 ventricles in larvae zebrafish. Thus, our results suggest that APP not only is distributed to cilia 273 but also seems to have an important function in ciliogenesis and brain development.

274 We used different antibodies to confirm the localization of App to the cilia. The punctate 275 localisation of APP indicates that the protein is randomly distributed within the cilium similar 276 to other membrane receptors such as SSTR3 and Smoothened (Smo) ${ }^{44,45}$. The distribution of 
APP within the plasma membrane varies between cell types, nonetheless a recent study suggested that APP clusters form groups of proteins within the plasma membrane ${ }^{46}$. The similarity with the punctate pattern found here suggests that App may form clusters within the cilium, at least in zebrafish and mice. In contrast, we observed a continuous rather than punctate distribution of APP in human ependymal cilia. Whether the observed differences are due to sample preparation or variations in APP distribution between species remains to be addressed. Moreover, the accumulation of App at the root of the basal body, as observed in the olfactory sensory neurons and otic vesicle cilia in larvae zebrafish, correlates with the findings reported by Yang and Li on APP enrichment along ciliary rootlets ${ }^{47}$.

The presence of APP within cilia raises the question of how APP is targeted to the cilium. The cilium membrane is continuous with the plasma membrane, yet it possesses a specific and conserved composition of proteins and lipids. This specification is considered to be established through an active transport of ciliary membrane proteins ${ }^{48}$ that at least partly depends on specific ciliary transport sequences (CTSs) within the proteins ${ }^{49}$. The presence of several such CTSs and their conservation between zebrafish, mice and human supports a motif-based transport of APP to cilia (Supplementary file 8). It will therefore be very interesting to address the extent to which these motifs are required for accumulation of APP at the root of the basal body and later distribution of APP out to the cilium.

APP expression by the ependymal cells was first reported in rodents and humans in the late 1980s and early $1990 \mathrm{~s}^{32,33,50,51}$. In line with these findings, our results not only confirm the expression of App in adult zebrafish ependymal cells, but in addition, show that APP localizes to ependymal motile cilia in vertebrates as far apart as zebrafish, mice, and humans. Our finding 
that loss of App results in morphologically abnormal ependymal cilia suggests a role of App in

302 ciliogenesis. However, the $a p p a^{-/} a p p b^{-/}$mutants gave rise to fertile adults without major phenotypic changes associated with cilia defects, such as curved body and hydrocephalus ${ }^{52-55}$.

304 In line with our findings, Olstad et al. recently reported that such phenotypes mainly associate 305 with primary cilia defects, while changes in motile cilia were more likely to result in ventricle 306 duct occlusion ${ }^{56}$. During early development, movement of cilia is a major factor maintaining

307 CSF flow within ventricles. Consequently, the cilia-driven flow is crucial to form and maintain 308 a proper brain ventricular system, as zebrafish, clawed frog and mouse ciliary mutants display 309 ventricular defects ${ }^{56}$. It is thus likely that the defective ventricle expansion observed in the $a p p a^{-/} a p p b^{-/-}$mutant larvae may result from changes in of motile cilia. Although we did not 311 observe a lack of diffusion between ventricles indicating duct occlusion, our data suggest that 312 App may be required in motile cilia to promote flow of CSF needed for ventricle formation 313 during early development ${ }^{56}$. It will thus be interesting to examine the extent to which cilia 314 movement and CSF flow change when altering App levels and at later developmental stages.

316 Our findings raise several questions regarding the role of APP in cilia and to which level cellular 317 processes associated with APP may be mediated through cilia. For example, the multi-ciliated 318 ependymal cell layer covering the brain ventricles is important for neurogenesis, both by 319 regulating the number of neural stem cells in the neurogenic niches of the subventricular zone

$320{ }^{51}$ and by facilitating the migration of new-born neuronal cells through cilia-regulated fluid 321 dynamics ${ }^{57}$. Interestingly, APP is also reported to regulate neurogenesis ${ }^{58-60}$ and to promote 322 neuronal migration ${ }^{9}$. Our understanding of APP-mediated processes continues to increase, but 323 the mechanisms by which these processes are orchestrated are yet not fully understood. 324 Therefore, the obvious overlap between functions mediated by ependymal cells and APP makes 
it tempting to speculate that APP might be important or at least partly required in ependymal cells to mediate cell migration and proliferation.

The length of cilia can be modified both by changes in the structural proteins involved in microtubule assembly but also depends on the cell proliferation and differentiation status, where proliferating cells generally have shorter cilia than growth arrested cells. As an example, modulation of several cell cycle-related kinases could alter ciliary length ${ }^{61}$. This is true for 332 primary cilia but to what extent this is valid for other types of cilia is not yet described. The mechanisms by which App contributes to regulate cilia length is beyond the scope of the present study but could potentially involve its role in cell differentiation ${ }^{6}$.

It is intriguing to think of APP in the cilium as a receptor that senses signalling molecules and metabolites transported through the ventricles by CSF. The hypothesis of APP acting as a receptor is supported by its similarities with type I membrane receptors and by the fact that the list of potential APP ligands continues to grow (review by ${ }^{6}$ ). Therefore, it is tempting to speculate that APP localized on the cilia interacts with CSF-circulating ligands, e.g., A $\beta$ peptides, growth factors, and hormones ${ }^{6,62}$, to mediate CSF-derived signalling.

342 Beyond the impact of App on ciliogenesis during development, it is intriguing to speculate on 343 the long-term effects of impaired near-wall CSF propulsion. This movement is thought to play 344 an important role in removal of waste products from the brain parenchyma ${ }^{63}$. Thus, it is likely 345 that subtle changes in the coordinated beating of cilia may contribute to altered regional CSF 346 flow that impairs clearance and hence contributes to a slow build-up of waste products over 347 time. In support are findings that individuals with Down syndrome, expressing approximately $34850 \%$ higher levels of APP, have changed CSF flow in the lateral ventricles ${ }^{64}$. Although the 
morphology of ependymal cilia of DS brains are unknown, in vitro cell cultures show decreased

350 primary cilia length ${ }^{26}$. Investigations addressing cilia morphology and function in the adult

351 zebrafish brain lacking App are ongoing in our lab; however, it will be equally important to

352 perform these experiments in app-knockout mice, as well as in mice overexpressing APP,

353 which results in altered post-translational processing of the protein.

The presence of APP in ependymal cells and their cilia also raises the question regarding their contribution to APP-derived fragments found in CSF. As at least some secretases needed for APP processing are present in cilia ${ }^{65}$, it is likely that the fragments detected in CSF not only originates from the brain parenchyma but also from APP being processed within the ependymal cells and the protruding cilia. The release of APP from ependymal cells could be mediated through the release of extracellular cleavage products or by budding extracellular vesicles and ectosomes. The latter process was in a recent study described as a common mechanism by which proteins are cleared from cilia instead of recycling by retrograde transport ${ }^{66}$. APPcontaining vesicles are released into the $\mathrm{CSF}^{67}$ and in a recent report, such microvesicles were

364 found to have lower levels of APP in AD patients compared to healthy individuals ${ }^{68}$. The impact of ependymal integrity and the contribution of cilia-mediated APP release need further studies but could potentially contribute to our interpretation of biomarkers used to assess 367 disease progression. Interestingly, a well-established feature of normal pressure hydrocephalus, 368 where ciliary function is impaired ${ }^{69}$, is decreased CSF levels of soluble APP and A $\beta$, which 369 are restored upon successful shunt treatment of the condition ${ }^{70-72}$.

371 While others have shown that APP and its processing machinery are expressed in the olfactory 
373 olfactory cilia in larvae zebrafish. Motile cilia of the olfactory sensory neurons (OSNs) in

374 zebrafish are essential to generate liquid flow in the nose pit to detect odorant molecules ${ }^{74}$. In

375 zebrafish, the olfactory epithelium can be divided into three categories of OSN, i.e., ciliated,

376 microvillus and crypt OSNs ${ }^{75,76}$. Each OSN expresses distinct classes of receptors and sensing

377 molecules and has a specific axonal pathway from the olfactory bulb leading towards higher

378 olfactory centres in either the telencephalon or the optic tectum. If APP is present in cilia of all

379 OSN or only a subset, needs to be confirmed. However, the presence of APP in the olfactory

380 cilia could potentially give clues on corresponding pathways and insights into the mechanisms

381 resulting in olfactory deficiencies in AD mouse models and neurodegenerative disease ${ }^{77}$.

382 Hearing is a major sensory input in vertebrates, which is known to decrease with aging. 383 Although the relationship between APP and hearing is less studied than many other areas, there are a few reports pointing to the loss of hearing associated with APP or its cleavage product A $\beta$ ${ }^{78-80}$. Our data, showing the presence of App in cilia mediating hearing, open up the possibility that nervous system-related changes in hearing may not only be due to defects in the brain regions receiving input from the auditory organ but also due to direct effects on the cilia. However, the function of App in the auditory system needs further investigation.

Altogether, our data show the presence of App in motile and non-motile cilia of the otic vesicle, 391 olfactory pit and ependymal cells lining the brain ventricles. We also report a conserved distribution, at least in the ependymal cilia, across vertebrates and that App is required for proper ciliogenesis and brain ventricle formation. The evolutionary conserved CTSs of APP and its expression throughout development and aging suggest a central role of APP within the ependyme. Further studies are required to fully understand the impact of App in cilia in our olfactory and auditory organs and to which extent defects in ependymal cell integrity and ciliation contribute to APP-related developmental processes and disease progression. 


\section{Methods}

399

400

401

402

403

404

405

406

407

408

409

410

411

412

413

414

415

416

417

418

419

420

421

\section{Animal care and ethics statement}

The zebrafish (Danio rerio) facilities and maintenance were approved and follow the guidelines of the Swedish National Board for Laboratory Animals. This study was approved by the Animal Ethical Committee at the University of Gothenburg. All procedures for the experiments were performed in accordance with the animal welfare guidelines of the Swedish National Board for Laboratory Animals and followed the recommendations in the ARRIVE guidelines ${ }^{81}$. Zebrafish were maintained in Aquatic Housing Systems (Aquaneering, San Diego, CA) at 28.5 ${ }^{\circ} \mathrm{C}$, under a 14:10 hour (h) light:dark cycle at the Institute of Neuroscience and Physiology, University of Gothenburg. Fish were fed twice daily a diet of live-hatched brine shrimps and Gemma fish food (Skretting, Amersfoort, Netherlands). System water was created using reverse osmosis water kept at a pH of 7.2-7.6 with $\mathrm{NaHCO}_{3}$ and coral sand and salt (Instant Ocean, Blacksburg, VA) to maintain the conductivity at $600 \mu \mathrm{S}$. Breeding of fish was carried out in 1$2 \mathrm{~L}$ breeding tanks and embryos were collected in embryo medium (EM) (1.0mM $\mathrm{MgSO}_{4}$, $0.15 \mathrm{mM} \mathrm{KH}_{2} \mathrm{PO}_{4}, 0.042 \mathrm{mM} \mathrm{Na}_{2} \mathrm{HPO}_{4}, 1 \mathrm{mM} \mathrm{CaCl}, 0.5 \mathrm{mM} \mathrm{KCl}, 15 \mathrm{mM} \mathrm{NaCl}, 0.7 \mathrm{mM}$ $\mathrm{NaHCO}_{3}$ ) and raised in a dark incubator at $28.5^{\circ} \mathrm{C}^{82}$.

The following fish lines were used in the present project; $\mathrm{AB}$ fish from the Zebrafish international resource centre (ZIRC) or was used for outbreeding and as wild-type background, $a p p b^{26 \_2-/ 7}$ and $a p p a^{-/}$as described below.

Human brain tissue samples were obtained from Queen Square Brain Bank for Neurological Disorders, Department of Clinical and Movement Neurosciences, Institute of Neurology, University College London (UCL). Ethical approval for the use of human post-mortem samples was approved by a London Research Ethics Committee and tissue stored for research under a license from the Human Tissue Authority with consent obtained from each donor. Human brain 
422 tissues were used in accordance with the Helsinki declaration and the regional ethics committees at UCL and the University of Gothenburg.

\section{Mutagenesis using the CRISPR/Cas9 system}

426 Genetic mutations in the appa gene were introduced using the CRISPR/Cas9 system as 427 previously described ${ }^{83}$. Briefly, gRNAs were generated with a target-specific DNA 428 oligonucleotide (Integrated DNA Technologies, Leuven, Belgium) containing a T7 promoter 429 sequence in the 5'-end and a 'generic' DNA oligonucleotide for the guide RNA. The two 430 oligonucleotides were annealed and extended with Platinum Taq DNA polymerase 431 (ThermoFisher, Waltham, MA), in a final concentration of $1 \mathrm{x}$ buffer, $0.25 \mathrm{mM} \mathrm{dNTP}, 0.5 \mu \mathrm{M}$ 432 of each oligonucleotide and $0.04 \mathrm{U} / \mathrm{ul}$ Taq with one cycle at the following temperatures $\left(98^{\circ} \mathrm{C}\right.$ $4332 \mathrm{~min} ; 50^{\circ} \mathrm{C} 10 \mathrm{~min}, 72^{\circ} \mathrm{C} 10 \mathrm{~min}$ ). The resulting product was analyzed on a $2.5 \%$ agarose 434 (Roche, Basel, Switzerland) gel to confirm a single fragment of 120 basepairs (bp) and used to 435 transcribe RNA. In vitro transcription was performed with the T7 Quick High Yield RNA 436 Synthesis kit (New England Biolabs, Ipswich, MA) and incubated at $37^{\circ} \mathrm{C}$ for $16 \mathrm{~h}$. DNA 437 template was removed with RNase Free DNase at $37^{\circ} \mathrm{C}$ for $15 \mathrm{~min}$. After purification with the 438 RNA clean \& concentrator-5 (Zymo Research, Irvine, CA), gRNA was analyzed on a 2.5\% 439 agarose gel for integrity and diluted to $250 \mu \mathrm{g} / \mu \mathrm{l}$ with $\mathrm{RNase}$ free water and stored at $-80^{\circ} \mathrm{C}$. 440 Cas9 protein was diluted to 500nM in Hepes (20mM HEPES, pH7.5; 150mM KCl) and stored 441 at $-80^{\circ} \mathrm{C}$. Embryos were co-injected with $50 \mathrm{pg}$ gRNA and $300 \mathrm{pg}$ Cas 9 protein at the one to 442 two cell stage using a microinjector apparatus FemtoJet ${ }^{\circledR}$ express (Eppendorf AG, Hamburg, 443 Germany). Injected embryos were screened for gRNA activity using the T7 endonuclease assay

444 (New England Biolabs, Ipswich, MA). Ten embryos from each gRNA injection were pooled at $44548 \mathrm{hpf}$ and genomic DNA extracted with $50 \mathrm{mM} \mathrm{NaOH}$ at $95^{\circ} \mathrm{C}$ for $30 \mathrm{~min}$. M13- and PIG446 tailed primers (IDT, Leuven, Belgium) were used to amplify a region surrounding the mutated 
447 site of each locus using $1 \mathrm{x}$ buffer, $2.5 \mathrm{mM} \mathrm{MgCl}_{2}, 0.2 \mathrm{mM} \mathrm{dNTP}, 0.2 \mu \mathrm{M}$ primers, $1 \mathrm{U}$ Taq 448 polymerase (Promega, Fitchburg, WI). The polymerase chain reaction (PCR) was purified on 449 an 1\% agarose gel with the QIAquick Gel Extraction Kit (Quiagen, Hilden, Germany) and then $450200 \mathrm{ng}$ of the purified PCR product was dissociated and reannealed $\left(95^{\circ} \mathrm{C}\right.$ for $5 \mathrm{~min}, 95-85^{\circ} \mathrm{C}$ 451 at $-2^{\circ} \mathrm{C} / \mathrm{s}, 85-25^{\circ} \mathrm{C}$ at $0.1^{\circ} \mathrm{C} / \mathrm{s}$ ) in a reaction containing $1 \mathrm{x}$ NEB buffer 2 (New England Biolabs, 452 Ipswich, MA) and then digested with 5U T7 endonuclease I (New England Biolabs, Ipswich, $453 \mathrm{MA}$ ) for one hour at $37^{\circ} \mathrm{C}$. Fragments were analyzed on a $2 \%$ agarose gel. The remaining 454 embryos were raised to adulthood and outcrossed with AB wild-type fish. Sixteen embryos 455 from each outcrossed pair were screened for mutations in the F1 generation using a three-primer 456 fluorescence PCR method. A 300-450 bp region surrounding the target site was amplified using 457 forward primers linked with a M13 sequence and a PIG-tailed reverse primer in combination 458 with a generic M13-FAM primer. The $a p p a^{C 21} 16$ mutants, refer to as $a p p a^{-/}$, carry a deletion 459 of $-10 \mathrm{bp}$ in exon 2. Sanger sequencing with BigDye ${ }^{\text {TM }}$ Terminator v1.1 Cycle Sequencing Kit 460 (Applied Biosystems ${ }^{\mathrm{TM}}$, Waltham, MA) on an ABI3130xl sequencer (SeqGen Inc, Los 461 Angeles, CA) revealed a deletion of ten nucleotides in exon 2 that likely introduce a frameshift 462 mutations. Heterozygous mutant carriers were raised and subsequently outcrossed into the wild463 type $\mathrm{AB}$ fish line until generation F4. Outcrossed adults were genotyped using M13-FAM 464 primers and PCR reactions diluted in $\mathrm{HiDi}^{\mathrm{TM}}$ formamide (Applied Biosystems ${ }^{\mathrm{TM}}$, Waltham, 465 MA) with $\mathrm{ROX}^{\mathrm{TM}} 500$ dye size ladder (ThermoFisher, Waltham, MA) and analyzed for 466 amplified fragment length polymorphism (AFLP) on an ABI3130x1 sequencer. Offspring from 467 heterozygous F4 inbreeds were inbred to generate homozygous wild-type and mutant lines. 468 Generation of $a p p a^{-/} a p p b^{-/}$double mutants were obtain from mating single mutant $a p p a^{-/-}$with 469 single mutant $a p p b^{-/}$. 
472 Sequences of APP were obtained from the UniProt database ${ }^{84}$ and aligned with ClustalW using 473 MegAlign Pro v17.2.1 (DNAstar, Inc., Madison, WI) The following sequences were used; 474 Homo sapiens APP751 (P05067-8), Mus musculus APP751 (P12023-3), Danio rerio Appa738 475 (Q90W28), Appb751 (B0V0E5). Amino acids conserved across all species were marked with 476 bright blue background.

\section{Whole-mount fluorescent in situ hybridization}

To detect appa and appb mRNA expression pattern in zebrafish larvae, fluorescent in situ

480 hybridization was performed. Antisense digoxigenin-labeled appa and appb RNA probes used 481 are described previously ${ }^{85}$. Zebrafish embryos were staged according to Kimmel et al. to the hours post-fertilization (hpf) ${ }^{86}$ and manually dechorionated with forceps (Dumont, Montignez, Switzerland). A treatment with 0.003\% PTU (1- phenyl-2-thiourea) (Sigma, St. Louis, MO) was performed around 23hpf stage to prevent pigmentation. Fluorescent in situ hybridization was performed as described by Lauter et al. ${ }^{87}$. Briefly, zebrafish larvae were euthanized in 0.2mg/ml ethyl 3-aminobenzoate methanesulfonate (tricaine) (MS-222, Sigma, St. Louis, MO) 82 and fixed at $30 \mathrm{hpf}$ in $4 \%$ paraformaldehyde (PFA) (Sigma, St. Louis, MO) for $24 \mathrm{~h}$ at $4{ }^{\circ} \mathrm{C}$. Embryos were washed in phosphate-buffered saline (PBS) with 0.1\% Tween-20 (PBST) and dehydrate into increasing methanol $(\mathrm{MeOH})$ gradients from 25 to $100 \%$. Embryos were incubated in $2 \%$ hydrogen peroxide $\left(\mathrm{H}_{2} \mathrm{O}_{2}\right)$ for $20 \mathrm{~min}$, then gradually rehydrated with decreasing $\mathrm{MeOH}$ gradients. Embryos were incubated in $10 \mu \mathrm{g} / \mathrm{ml}$ proteinase $\mathrm{K}$ (in $10 \mathrm{mM}$ Tris$\mathrm{HCl} \mathrm{pH}$ 8.0, $1.0 \mathrm{mM}$ EDTA) for $10 \mathrm{~min}$ at room temperature (RT). The reaction was stopped with $2 \mathrm{mg} / \mathrm{ml}$ glycine in PBST and then the embryos were postfix in $4.0 \%$ PFA for $20 \mathrm{~min}$. PBST washes were performed before incubation in prehybridization buffer (HB; 50\% deionized formamide, 5x saline-sodium citrate (SSC) (3M NaCl, $300 \mathrm{mM}$ tri-sodium citrate, $\mathrm{pH}$ 7.0), 5 $\mathrm{mg} / \mathrm{ml}$ torula RNA (Sigma, St. Louis, MO), $50 \mu \mathrm{g} / \mathrm{ml}$ heparin sodium salt and $0.1 \%$ Tween- 
20). Embryos were pre-hybridized at $70^{\circ} \mathrm{C}$ for $1 \mathrm{~h}$. Then, hybridization was done with

498 selectively $50 \mathrm{ng}$ of DIG-labelled appa or appb RNA in HB with 5\% dextran sulfate (Sigma,

499 St. Louis, MO) at $70{ }^{\circ} \mathrm{C}$ overnight. The next day, embryos were washed in warm SSC with

$500 \quad 0.1 \%$ Tween-20 followed by PBST only. After that, a 1h-blocking incubation at RT in PBST

501 with $8 \%$ goat serum (Sigma, St. Louis, MO) was performed. For the antibody treatment, a

502 sheep-anti-digoxigenin-peroxidase (POD)-Fab fragments antibody (1:500 in blocking solution)

503 (Roche, Basel, Switzerland) was used and embryos were incubated in the dark overnight at $4^{\circ} \mathrm{C}$,

504 without agitation. To remove excess antibody, embryos were then washed in PBST at RT in

505 gentle agitation. To amplify the signal, tyramide signal amplification (TSA) was used by

506 combining 5-carboxyfluorescein succinimidyl ester (Molecular Probes, Eugene, OR) with

507 tyramine hydrochlorine (Sigma, St. Louis, MO) at a 1.1:1 respective equimolar ratio. Vanillin

$508(0.45 \mathrm{mg} / \mathrm{mL})($ Sigma, St. Louis, MO) was used as a POD accelerator and diluted in borate

509 buffer $\mathrm{pH}$ 8.5. Embryos were incubated with the TSA and POD accelerator reaction in the dark

510 without agitation for $15 \mathrm{~min}$ at RT. To stop the TSA reaction, embryos were washed in PBST

511 and then incubate in $100 \mathrm{mM}$ glycine- $\mathrm{HCl} \mathrm{pH} 2.0$ to inactivate the POD reaction followed by

512 additional PBST washing. To avoid shrinkage, embryos were then incubated in an increasing

513 glycerol gradient (in PBST, 40mM NaHCO 3 ). Whole embryos were mounted on glass bottom

$51435 \mathrm{~mm}$ Petri dish (Cellvis, Mountain View, CA) in 1\% low-melting agarose (Sigma, St. Louis,

515 MO). Samples were imaged as stacks using inverted Nikon A1 confocal system (Nikon

516 Instruments, Melville, NY) using a 20x objective (Plan-Apochromat 20x/0,75) and 40x water-

517 immersion objective (Apochromat LWD 40x/1,15). Image processing was done using ImageJ

518 FIJI software (NIH, Bethesda, MD). 
522 To detect protein expression, immunofluorescence experiments were performed in wholemount AB zebrafish larvae. A treatment with $0.003 \%$ PTU was performed around 23hpf stage to prevent pigmentation. Then freshly euthanized embryos were fixed at $30 \mathrm{hpf}$ for $2 \mathrm{~h}$ in $4 \%$ PFA at RT on slow agitation. After fixation, embryos were washed with PBS with $0.5 \%$ Triton$\mathrm{X}$ (PBTx) at RT. Followed up by incubation in blocking solution (5\% goat serum donor herd (GS) (Sigma, St. Louis, MO), 2\% bovine serum albumin (BSA) (Sigma, St. Louis, MO) , $1 \%$ DMSO (Sigma, St. Louis, MO) and 0.5\% PBTx) for 3h at RT. The larvae were then incubated overnight at $4^{\circ} \mathrm{C}$ on slow agitation with the desired primary antibodies in blocking solution:

530 mouse IgG2b anti-acetylated tubulin monoclonal antibody (1:1000) (Sigma, St. Louis, MO), recombinant rabbit anti-amyloid precursor protein monoclonal antibody Y188 (1:500) (Abcam,

532 Cambridge, United Kingdom), and/or mouse anti-glutamylated tubulin monoclonal antibody 533 (1:1000) (Adipogen, San Diego, CA). The zebrafish larvae used for negative control were 534 incubated in blocking solution only. The next day, embryos were washed (5x 45min) with 535 PBSTx at RT and incubated in dark with the specific secondary antibodies overnight at $4{ }^{\circ} \mathrm{C}$, in 536 blocking solution: goat anti-mouse IgG2b Alexa Fluor-647 (1:1000) (Invitrogen Thermo 537 Fisher, Waltham, MA) and goat anti-rabbit IgG Alexa Fluor-488 (1:1000) (Invitrogen Thermo 538 Fisher, Waltham, MA), or goat anti-mouse IgG1 Alexa Fluor-568 (1:1000) (Invitrogen Thermo 539 Fisher, Waltham, MA). The zebrafish larvae used for negative control were also incubated with 540 the former secondary antibodies. The larvae were then washed with PBTx at RT and incubated 541 for 15 min with DAPI (1:1000) (ThermoFisher, Waltham, MA) to stain the nuclei in PBS at RT 542 before the final washes. Stained larvae were mounted in $1 \%$ low-melting point agarose, on glass 543 bottom $35 \mathrm{~mm}$ Petri dish. 
545 Brains from adult zebrafish (AB, 2 year-old) and mouse (C57B16/n, 8-9 week-old). Brains were 546 fixed in $4 \%$ PFA in PBS overnight at $4{ }^{\circ} \mathrm{C}$ and then washed and immersed in $30 \%$ sucrose 547 solution in PBS, after which they were frozen in OCT cryomount (Histolab, Askim, Sweden). 548 Coronal or sagittal cryosections from adult zebrafish $(25 \mu \mathrm{m})$ and mouse brains $(16 \mu \mathrm{m})$ slices 549 were stored at $-80^{\circ} \mathrm{C}$ prior to use. Sections were air dried for $15 \mathrm{~min}$ at RT then rehydrated in 550 PBS. Slices were permeabilized in $0.1 \%$ PBTx for 10 min at RT and washed 3x in PBS for 551 15min each. A 0.1\% Sudan Black B (SBB) (Sigma, St. Louis, MO) in 70\% EtOH treatment 552 was performed for $20 \mathrm{~min}$ at RT. Slides were then washed in PBS for 3x5 min. The slides were then incubated in blocking solution of $2 \%$ GS in PBS at RT for $1 \mathrm{~h}$, followed by the incubation with the primary antibodies in $2 \% \mathrm{BSA}$ at $4{ }^{\circ} \mathrm{C}$ overnight: mouse IgG2b anti-acetylated tubulin monoclonal antibody $(1: 1000)$, recombinant rabbit anti-amyloid precursor protein monoclonal antibody (Y188) (1:500) or mouse anti-amyloid precursor protein A4 antibody (clone 22C11) (1:500) (Merck Millipore, Burlington, MA), or rabbit IgG (1:500) (Abcam, Cambridge, United Kingdom) and/or with blocking solution only for negative controls. The next day, slides were wash $3 \mathrm{x}$ in PBS for $15 \mathrm{~min}$ each and incubated with the secondary antibody in $2 \%$ BSA at RT 560 for 3.5h combined with DAPI (1:1000): goat anti-mouse IgG2b Alexa Fluor-647 (1:1000) 561 and/or goat-anti rabbit Alexa Fluor-488 (1:1000) and/or goat anti-mouse IgG1 Alexa Fluor-488 562 (1:1000) (ThermoFisher, Waltham, MA) and/or goat anti-mouse IgG1 Alexa Fluor-568 (1:1000). The slides were then washed $3 \times 15 \mathrm{~min}$ in PBS and shortly rinsed in $\mathrm{ddH}_{2} \mathrm{O}$ to remove 564 any residual salts. The slides were covered with coverslips using ProLong gold antifade 565 mounting medium (Invitrogen Thermo Fisher, Waltham, MA).

566 Samples were imaged using Zeiss LSM710 inverted confocal microscope (Carl-Zeiss, Jena, 567 Germany) using 40x water immersion objective (Plan-Apochromat 40x/1.0) and a 63x oil568 immersion objective (Plan-Apochromat 40x/1.0) or with Zeiss LSM880 Airyscan inverted 569 confocal microscope (Carl-Zeiss, Jena, Germany) using 40x water immersion objective (LCD- 
Apochromat 40x/1.0) and 63x oil-immersion objective (Plan-Apochromat 63x/1.4). Image

571 processing and intensity profiles were performed with ImageJ FIJI program.

572

573

574

575

576

\section{Human brain sections immunofluorescent staining}

Neurologically normal human post-mortem control tissue was obtained from Queen Square Brain Bank for Neurological Studies. Paraffin-embedded sections were cut from caudate nucleus brain region, which contains ependymal lining containing cilia. Sections were dewaxed in three changes of xylene and rehydrated using graded alcohols. Endogenous peroxidase activity was blocked using $0.3 \% \mathrm{H}_{2} \mathrm{O}_{2}$ in $\mathrm{MeOH}$ for 10 min followed by pressure cooker pretreatment for $10 \mathrm{~min}$ in citrate buffer, $\mathrm{pH}$ 6.0. Non-specific binding was blocked using $10 \%$ non-fat dried milk (Sigma-Aldrich, St. Louis, MO) in Tris buffered saline-Tween (TBS-Tween) before incubating with either anti-acetylated tubulin (1:1000) or anti-APP (1:500) antibodies at RT for $1 \mathrm{~h}$. A biotinylated mouse anti-rabbit IgG antibody (1:200) (Agilent DAKO, Glostrup, Denmark) was added for a 30 min incubation with the sections at RT followed by avidin-biotin complex (Vector Laboratories, Burlingame, CA). Coloration was developed with diaminobenzidine (Sigma-Aldrich, St. Louis, $\mathrm{MO}$ ) activated with $\mathrm{H}_{2} \mathrm{O}_{2}{ }^{88}$.

\section{Protein extraction from whole zebrafish larvae and western blotting}

Protein was extracted from 3dpf double $a p p a^{-/} a p p b^{-/}$mutant whole larvae (60 larvae per n, n=3) to confirm loss of protein. Larvae were euthanized, deyolked with ice-cold PBS and snap frozen in liquid nitrogen prior to use and stored at $-80^{\circ} \mathrm{C}$. Samples were homogenized in an icecold lysis buffer (10 mM Tris- $\mathrm{HCl} \mathrm{pH}$ 8.0, 2\% sodium deoxycholate, 2\% SDS, $1 \mathrm{mM}$ EDTA, $0.5 \mathrm{M} \mathrm{NaCl}, 15 \%$ glycerol) supplemented with protease inhibitors cocktail (Roche, Basel, Switzerland) and using glass tissue grinder, on ice. Samples were then incubated $20 \mathrm{~min}$ on ice, 
594 sonicated for $10 \mathrm{~min}$ on max level and centrifuged at $10,000 \times \mathrm{g}$ at $4^{\circ} \mathrm{C}$. Supernatants were 595 collected and kept on ice and protein concentration measured with a BCA Protein Assay Kit 596 (ThermoFisher, Waltham, MA) and samples stored at $-80^{\circ} \mathrm{C}$. Proteins samples (40-60ug) were 597 then diluted in a denaturing lysis buffer (1X NuPAGE ${ }^{\circledR}$ LDS Sample Buffer (ThermoFisher, 598 Waltham, MA), 0.05M DTT (Sigma-Aldrich, St. Louis, MO), lysis buffer completed with 599 protease inhibitors) and then boiled for $5 \mathrm{~min}$ at $95^{\circ} \mathrm{C}$. Proteins were then separated on a 600 NuPAGE $^{\circledR}$ NOVEX $^{\circledR}$ Bis-TRIS pre-cast gel (Invitrogen Thermo Fisher, Waltham, MA) and 601 transferred onto a $0.2 \mu \mathrm{m}$ nitrocellulose membrane (GE Healthcare, Chicago, IL). The 602 membrane was incubated in a blocking solution (5\% milk) for $2 \mathrm{~h}$ at RT and then immunoblotted 603 with the desired primary antibodies overnight at $4^{\circ} \mathrm{C}$ : rabbit anti-amyloid precursor protein 604 monoclonal antibody (Y188) (1:2000) or mouse anti-amyloid precursor protein A4 antibody 605 (clone 22C11) (1:5000) and with a loading concentration control mouse anti-GAPDH-HRP 606 conjugated (1:20000) (Novus Biologicals, Centennial, CO) or mouse anti- $\alpha$-tubulin monoclonal

607 (1:10000) (Sigma, St. Louis, MO). The membrane was then washed in TBS-Tween 3x 10min 608 at RT and incubated with the secondary antibodies anti-rabbit-HRP (1:5000) (Cell Signaling, 609 Danvers, MA) for $1 \mathrm{~h}$ at RT. The membrane was washed 3x10min in TBS-Tween before being 610 developed. The signal was developed using SuperSignal West Dura Extended Duration 611 Substrate kit (ThermoFisher, Waltham, MA) and imaged using ChemiDoc Imaging (Bio-Rad, 612 Hercules, CA). Western blot images were processed and analysed using Image Lab program 613 (Bio-Rad, Hercules, CA). Quantification of band intensities were performed by Image Lab 614 (Bio-Rad, Hercules, CA) with GAPDH or alpha-tubulin used to control protein loading. 615 Samples were normalized to controls. 
618 To confirm appa and appb mRNA levels decrease in our double mutant ( $\left.a p p a^{-/} a p p b^{-/}\right)$, RNA

619 was extracted from $24 \mathrm{hpf}$ whole larvae (10 larvae per $\mathrm{n}, \mathrm{n}=5$ ). Total RNA was extracted using

620 TRI Reagent ${ }^{\circledR}$ (Sigma, St. Louis, MO). Then, RNA samples were treated with RQ1 RNase-free

621 DNase 1x reaction buffer and RQ1 RNase-free DNase (Promega, Fitchburg, WI). cDNA was

622 synthesized using High-Capacity RNA-to-cDNA ${ }^{\mathrm{TM}}$ Kit (Applied Biosystems ${ }^{\mathrm{TM}}$, Waltham, 623 MA) with RNase inhibitor and converted in a single-cycle reaction on a 2720 Thermal Cycler 624 (Applied Biosystems ${ }^{\mathrm{TM}}$, Waltham, MA). Quantitative PCR was performed with inventoried TaqMan Gene Expression Assays with FAM reporter dye in TaqMan Universal PCR Master

626 Mix with UNG (ThermoFisher, Waltham, MA). The assay was carried out on Micro-Amp 96627 well optical microtiter plates (ThermoFisher, Waltham, MA) on a 7900HT Fast QPCR System 628 (Applied Biosystems ${ }^{\mathrm{TM}}$, Waltham, MA). qPCR results were analysed with the SDS 2.3 software 629 (Applied Biosystems ${ }^{\mathrm{TM}}$, Waltham, MA). cDNA values from each sample was normalized with 630 average $\mathrm{C}_{\mathrm{T}}$ 's of house-keeping genes (eeflall1 and actb1), then the relative quantity was 631 determined using the $\Delta \Delta \mathrm{C}_{\mathrm{T}}$ method ${ }^{89}$ with the sample of wild-type sibling embryos (24 hpf) as 632 the calibrator. TaqMan ${ }^{\circledR}$ Gene Expression Assays (Applied Biosystems ${ }^{\mathrm{TM}}$, Waltham, MA) were 633 used for the following genes: amyloid beta (A4) precursor protein A (appa) (Dr03144365_m1), 634 eukaryotic translation elongation factor 1 alpha 1, like 1 (eefla1l1) (Dr03432748_m1) and 635 actin, beta 1 (actb1) (Dr03432610_m1).

\section{Cilia length measurement in zebrafish larvae}

638 To compare the number of brain ependymal cilia and their length, 30 hpf AB wild-type and $639 \mathrm{appa}^{-/} \mathrm{appb}^{-/}$zebrafish larvae were used. The larvae were treated with PTU, fixed in 4\% PFA 640 and the immunostaining with antibody against acetylated tubulin was performed as describe in 641 the section above. Stacks (of around $25 \mu \mathrm{m}$ depending on the angle of the mounted sample) were 642 taken in the region of interest (ROI) of the dorsal portion of the diencephalic ventricle using 
643 Zeiss LSM710 confocal microscope using inverted 40x water immersion objective (Plan-

644 Apochromat 40x/1.0). Images were then processed using Imaris (BITPLANE ${ }^{\mathrm{TM}}$, Belfast, 645 United Kingdom) and the cilia length was measured with the acetylated tubulin signal using the 646 "measuring points" tool of the program. Raw data of the measurement were exported to 647 Microsoft Excel and compiled into GraphPad Prism ${ }^{\circledR} 7$ for statistical analysis.

\section{Brain ventricles injection and size measurement}

649 To measure the size of the brain ventricles in live zebrafish, 2dpf PTU-treated zebrafish larvae 650 were used. Rhodamine-Dextran injection protocol was performed as describe by Gutzman and 651 Sive ${ }^{90}$. Briefly, the larvae were anesthetized with tricaine in the EM and transferred onto a Petri 652 dish covered with $1 \%$ agarose, lined with rows moulded. The larvae were kept in EM 653 complemented with tricaine during the whole procedure and place on a ventral position, with 654 top of their head facing upwards. Injections were performed using borosilicate injection needles previously pulled (P-97 Flaming/Brown micropipette puller) (Sutter Instrument, Novato, CA).

656 Using a microinjector apparatus, 2nl of Rhodamine B isothiocyanate-Dextran (Sigma, St. 657 Louis, MO) were injected in the hindbrain ventricle without perforating or hitting the brain 658 tissue below.

659 Larvae with non-effective injections were sorted out using a fluorescent stereomicroscope 660 (Nikon Instruments, Melville, NY). Quickly after the sorting, the larvae were mounted in 1\% 661 low-melting point agarose on glass bottom $35 \mathrm{~mm}$ Petri dish. Confocal imaging stacks were 662 acquired using an inverted Nikon A1 confocal system using a 20x objective (Plan-Apochromat $66320 x / 0,75)$. Image processing of the confocal stacks were done with Imaris program. The 664 "surface" tool option was used for each sample. Data of the surface volume and area were 665 automatically generated by the program. Length measurements of the areas of the ventricles 666 were obtain manually with the "measuring tool". All data were exported into Microsoft Excel 667 and GraphPad® 7 Prism for statistical analysis. 
669 To evaluate the integrity of the internal structure of the axonemes and microtubules doublets of 670 the brain motile cilia in older zebrafish, transmission electron microscopy was performed on 671 fixed brains. Adult zebrafish were euthanized in tricaine and brains dissected, rinsed in ice-cold 672 PBS and fixed in 2\% PFA and 2\% glutaraldehyde (Sigma, St. Louis, MO), in 0.042M Millonig 673 buffer $\left(0.081 \mathrm{M} \mathrm{Na}_{2} \mathrm{HPO}_{4}, 0.0183 \mathrm{M} \mathrm{NaH}_{2} \mathrm{PO}_{4}, 0.086 \mathrm{M} \mathrm{NaCl}\right) \mathrm{pH} 7.4$ at least $24 \mathrm{~h}$ at $4^{\circ} \mathrm{C}$. After 674 fixation, brains were cut in two halves and then treated in 2\% osmium tetroxide (Sigma, St. 675 Louis, MO) in 0.1M Millonig buffer $\mathrm{pH}$ 7.4. Specimens were then rinsed and incubated 676 overnight in $4 \%$ sucrose solution in $0.1 \mathrm{M}$ Millonig buffer $\mathrm{pH} 7.4$ after which they were 677 dehydrated in series of ethanol and embedded in a mix of acetone and agar 100 resin plastic 678 (TAAB Laboratories Equipment Ltd, Berks, United Kingdom) and allowed to polymerize for $67948 \mathrm{~h}$. Blocks were trimmed as semi-thin $(1 \mu \mathrm{m})$ and ultra-thin $(70 \mathrm{~nm})$ sections collected with a 680 commercial ultramicrotome (Leica EM UC7, Leica Microsystems, Wetzlar, Germany). 681 Sections were post-stained with 5\% uranyl acetate in distilled $\mathrm{H}_{2} \mathrm{O}$ during 40-60 min, rinsed in 682 distilled $\mathrm{H}_{2} \mathrm{O}$ and then treated with $0.3 \%$ Lead Citrate (ThermoFisher, Waltham, MA) for 3068360 s. Images were acquired using secondary electron detection. Images were acquired with a 684 Tecnai Spirit BT transmission electron microscope (Field Electron and Ion Company, 685 Hillsboro, OR).

\section{Statistical analysis}

688 Statistical analysis was performed using GraphPad 7 software (Prism ${ }^{\circledR}$, San Diego, CA). Data 689 were presented as means with standard deviation $( \pm$ SD) or standard errors of the mean $( \pm$ 690 SEM). For analysis of cilia length, D’Agostino \& Pearson normality test $(\mathrm{P}<0.0001)$ and non691 parametric two-tailed Mann-Whitney U tests were performed. Results related to qPCR and 692 western blot quantification, and ventricle size measurements were compared statistically using 
693

694

695

696

697

698

699

700

701

702

703

704

705

706

707

708

709

710

711

712

713

714

715

716

717

718

719

720

721

722

723

724

725

726

727

728

729

730

731

unpaired Student's t-tests. Statistical significance was set at $\rho<0.05(*), 0.01(* *), 0.005(* * *)$ and $0.0001(* * * *)$.

\section{Data availability}

The datasets generated during and/or analysed during the current study are available from the corresponding author on reasonable request.

\section{References}

1 Selkoe, D. J. \& Hardy, J. The amyloid hypothesis of Alzheimer's disease at 25 years. EMBO Mol Med 8, 595-608, doi:10.15252/emmm.201606210 (2016).

2 Blennow, K., de Leon, M. J. \& Zetterberg, H. Alzheimer's disease. The Lancet 368, 387403, doi:10.1016/s0140-6736(06)69113-7 (2006).

3 Wang, B. et al. The Amyloid Precursor Protein Controls Adult Hippocampal Neurogenesis through GABAergic Interneurons. Journal of Neuroscience 34, 1331413325, doi:10.1523/jneurosci.2848-14.2014 (2014).

4 Caille, I. et al. Soluble form of amyloid precursor protein regulates proliferation of progenitors in the adult subventricular zone. Development 131, 2173-2181, doi:10.1242/dev.01103 (2004).

5 Young-Pearse, T. L., Chen, A. C., Chang, R., Marquez, C. \& Selkoe, D. J. Secreted APP regulates the function of full-length APP in neurite outgrowth through interaction with integrin beta1. Neural Dev 3, 15, doi:10.1186/1749-8104-3-15 (2008).

6 Deyts, C., Thinakaran, G. \& Parent, A. T. APP Receptor? To Be or Not To Be. Trends Pharmacol Sci 37, 390-411, doi:10.1016/j.tips.2016.01.005 (2016).

7 Banote, R. K. et al. Amyloid precursor protein-b facilitates cell adhesion during early development in zebrafish. Sci Rep 10, 10127, doi:10.1038/s41598-020-66584-8 (2020).

8 Wang, Z. et al. Presynaptic and postsynaptic interaction of the amyloid precursor protein promotes peripheral and central synaptogenesis. J Neurosci 29, 10788-10801, doi:10.1523/JNEUROSCI.2132-09.2009 (2009).

9 Young-Pearse, T. L. et al. A Critical Function for -Amyloid Precursor Protein in Neuronal Migration Revealed by In Utero RNA Interference. Journal of Neuroscience 27, 14459-14469, doi:10.1523/jneurosci.4701-07.2007 (2007).

10 Muller, U. C., Deller, T. \& Korte, M. Not just amyloid: physiological functions of the amyloid precursor protein family. Nat Rev Neurosci 18, 281-298, doi:10.1038/nrn.2017.29 (2017).

11 Haass, C., Kaether, C., Thinakaran, G. \& Sisodia, S. Trafficking and proteolytic processing of APP. Cold Spring Harb Perspect Med 2, a006270, doi:10.1101/cshperspect.a006270 (2012).

12 Brown, J. M. \& Witman, G. B. Cilia and Diseases. Bioscience 64, 1126-1137, doi:10.1093/biosci/biu174 (2014). 
13 Park, S. M., Jang, H. J. \& Lee, J. H. Roles of Primary Cilia in the Developing Brain. Front Cell Neurosci 13, 218, doi:10.3389/fncel.2019.00218 (2019).

14 Spassky, N. et al. Adult ependymal cells are postmitotic and are derived from radial glial cells during embryogenesis. $J$ Neurosci 25, 10-18, doi:10.1523/JNEUROSCI.1108-04.2005 (2005).

15 Lechtreck, K. F., Delmotte, P., Robinson, M. L., Sanderson, M. J. \& Witman, G. B. Mutations in Hydin impair ciliary motility in mice. J Cell Biol 180, 633-643, doi:10.1083/jcb.200710162 (2008).

Abbott, N. J., Pizzo, M. E., Preston, J. E., Janigro, D. \& Thorne, R. G. The role of brain barriers in fluid movement in the CNS: is there a 'glymphatic' system? Acta Neuropathol 135, 387-407, doi:10.1007/s00401-018-1812-4 (2018).

17 Ethell, D. W. Disruption of cerebrospinal fluid flow through the olfactory system may contribute to Alzheimer's disease pathogenesis. J Alzheimers Dis 41, 1021-1030, doi:10.3233/JAD-130659 (2014).

18 Kapoor, K. G., Katz, S. E., Grzybowski, D. M. \& Lubow, M. Cerebrospinal fluid outflow: an evolving perspective. Brain Res Bull 77, 327-334, doi:10.1016/j.brainresbull.2008.08.009 (2008).

19 Klein, S. et al. Truncating mutations in APP cause a distinct neurological phenotype. Ann Neurol 80, 456-460, doi:10.1002/ana.24727 (2016).

20 Kiprilov, E. N. et al. Human embryonic stem cells in culture possess primary cilia with hedgehog signaling machinery. J Cell Biol 180, 897-904, doi:10.1083/jcb.200706028 (2008).

21 Magara, F. et al. Genetic background changes the pattern of forebrain commissure defects in transgenic mice underexpressing the -amyloid-precursor protein. Proceedings of the National Academy of Sciences 96, 4656-4661, doi:10.1073/pnas.96.8.4656 (1999).

22 Baudoin, J. P. et al. Tangentially migrating neurons assemble a primary cilium that promotes their reorientation to the cortical plate. Neuron 76, 1108-1122, doi:10.1016/j.neuron.2012.10.027 (2012).

23 Guo, J. et al. Primary Cilia Signaling Shapes the Development of Interneuronal Connectivity. Dev Cell 42, 286-300 e284, doi:10.1016/j.devcel.2017.07.010 (2017).

24 Higginbotham, H. et al. Arl13b-regulated cilia activities are essential for polarized radial glial scaffold formation. Nat Neurosci 16, 1000-1007, doi:10.1038/nn.3451 (2013).

25 Guo, J. et al. Primary Cilia Signaling Promotes Axonal Tract Development and Is Disrupted in Joubert Syndrome-Related Disorders Models. Developmental Cell 51, 759774.e755, doi:10.1016/j.devcel.2019.11.005 (2019).

26 Galati, D. F., Sullivan, K. D., Pham, A. T., Espinosa, J. M. \& Pearson, C. G. Trisomy 21 Represses Cilia Formation and Function. Dev Cell 46, 641-650 e646, doi:10.1016/j.devcel.2018.07.008 (2018).

27 Chakravarthy, B. et al. Reduction of the immunostainable length of the hippocampal dentate granule cells' primary cilia in 3xAD-transgenic mice producing human A $\beta 1-42$ and tau. Biochemical and Biophysical Research Communications 427, 218-222, doi:10.1016/j.bbrc.2012.09.056 (2012).

28 Ibanez-Tallon, I. et al. Dysfunction of axonemal dynein heavy chain Mdnah5 inhibits ependymal flow and reveals a novel mechanism for hydrocephalus formation. Hum Mol Genet 13, 2133-2141, doi:10.1093/hmg/ddh219 (2004).

29 Vorobyeva, A. G. \& Saunders, A. J. Amyloid-beta interrupts canonical Sonic hedgehog signaling by distorting primary cilia structure. Cilia 7, 5, doi:10.1186/s13630-018-0059y (2018). 
30 Musa, A., Lehrach, H. \& Russo, V. A. Distinct expression patterns of two zebrafish homologues of the human APP gene during embryonic development. Dev Genes Evol 211, 563-567, doi:10.1007/s00427-001-0189-9 (2001).

31 Tanimoto, M., Ota, Y., Inoue, M. \& Oda, Y. Origin of inner ear hair cells: morphological and functional differentiation from ciliary cells into hair cells in zebrafish inner ear. J Neurosci 31, 3784-3794, doi:10.1523/JNEUROSCI.5554-10.2011 (2011).

32 Kawarabayashi, T., Shoji, M., Harigaya, Y., Yamaguchi, H. \& Hirai, S. Amyloid beta/A4 protein precursor is widely distributed in both the central and peripheral nervous systems of the mouse. Brain Res 552, 1-7, doi:10.1016/0006-8993(91)90651b (1991).

792

793

794

795

796

797

798

799

800

Ohta, M. et al. Immunohistochemical distribution of amyloid precursor protein during normal rat development. Brain Res Dev Brain Res 75, 151-161, doi:10.1016/01653806(93)90019-7 (1993).

34 Stern, R. A., Otvos, L., Jr., Trojanowski, J. Q. \& Lee, V. M. Monoclonal antibodies to a synthetic peptide homologous with the first 28 amino acids of Alzheimer's disease beta-protein recognize amyloid and diverse glial and neuronal cell types in the central nervous system. Am J Pathol 134, 973-978 (1989).

801

802

803

804

805

806

807

Fame, R. M., Chang, J. T., Hong, A., Aponte-Santiago, N. A. \& Sive, H. Directional cerebrospinal fluid movement between brain ventricles in larval zebrafish. Fluids Barriers CNS 13, 11, doi:10.1186/s12987-016-0036-z (2016).

36 Manton I.; Clarke, B. An electron microscope study of the spermatozoid of sphagnum. Journal of Experimental Botany 3, 265-275 (1952).

37 Satir, P. Studies on cilia: II. Examination of the distal region of the ciliary shaft and the role of the filaments in motility. Journal of Cell Biology, 805-834 (1965).

38 Satir, P., Heuser, T. \& Sale, W. S. A Structural Basis for How Motile Cilia Beat. Bioscience 64, 1073-1083, doi:10.1093/biosci/biu180 (2014).

809

39 Tam, B. M., Moritz, O. L., Hurd, L. B. \& Papermaster, D. S. Identification of an outer segment targeting signal in the $\mathrm{COOH}$ terminus of rhodopsin using transgenic Xenopus laevis. J Cell Biol 151, 1369-1380, doi:10.1083/jcb.151.7.1369 (2000).

40 Deretic, D. A role for rhodopsin in a signal transduction cascade that regulates membrane trafficking and photoreceptor polarity. Vision Research 46, 4427-4433, doi:10.1016/j.visres.2006.07.028 (2006).

41 Rakoczy, E. P., Kiel, C., McKeone, R., Stricher, F. \& Serrano, L. Analysis of diseaselinked rhodopsin mutations based on structure, function, and protein stability calculations. J Mol Biol 405, 584-606, doi:10.1016/j.jmb.2010.11.003 (2011).

817

818

819

820

821

822

823

824

825

826

827

Berbari, N. F., Johnson, A. D., Lewis, J. S., Askwith, C. C. \& Mykytyn, K. Identification of ciliary localization sequences within the third intracellular loop of $\mathrm{G}$ protein-coupled receptors. Mol Biol Cell 19, 1540-1547, doi:10.1091/mbc.E07-09-0942 (2008).

43 Domire, J. S. et al. Dopamine receptor 1 localizes to neuronal cilia in a dynamic process that requires the Bardet-Biedl syndrome proteins. Cell Mol Life Sci 68, 2951-2960, doi:10.1007/s00018-010-0603-4 (2011).

44 Chakravarthy, B. et al. The p75 neurotrophin receptor is localized to primary cilia in adult murine hippocampal dentate gyrus granule cells. Biochem Biophys Res Commun 401, 458-462, doi:10.1016/j.bbrc.2010.09.081 (2010).

45 Ye, F. et al. Single molecule imaging reveals a major role for diffusion in the exploration of ciliary space by signaling receptors. eLife 2, doi:10.7554/elife.00654 (2013). 
46 de Coninck, D., Schmidt, T. H., Schloetel, J. G. \& Lang, T. Packing Density of the

830

831

832

833

834

835

836

837

838

839

840

841

842

843

844

845

846

847

848

849

850

851

852

853

854

855

856

857

858

859

860

861

862

863

864

865

866

867

868

869

870

871

872

873

874

875

876

877

878

Amyloid Precursor Protein in the Cell Membrane. Biophys J 114, 1128-1141, doi:10.1016/j.bpj.2018.01.009 (2018).

47 Yang, J. \& Li, T. The ciliary rootlet interacts with kinesin light chains and may provide a scaffold for kinesin-1 vesicular cargos. Exp Cell Res 309, 379-389, doi:10.1016/j.yexcr.2005.05.026 (2005).

48 Long, H. \& Huang, K. Transport of Ciliary Membrane Proteins. Frontiers in Cell and Developmental Biology 7, doi:10.3389/fcell.2019.00381 (2020).

49 Malicki, J. \& Avidor-Reiss, T. From the cytoplasm into the cilium: bon voyage. Organogenesis 10, 138-157, doi:10.4161/org.29055 (2014).

50 Mita, S., Schon, E. A. \& Herbert, J. Widespread expression of amyloid beta-protein precursor gene in rat brain. Am J Pathol 134, 1253-1261 (1989).

51 Alvarez-Buylla, A. \& Lim, D. A. For the long run: maintaining germinal niches in the adult brain. Neuron 41, 683-686, doi:10.1016/s0896-6273(04)00111-4 (2004).

52 Zhang, X. et al. Cilia-driven cerebrospinal fluid flow directs expression of urotensin neuropeptides to straighten the vertebrate body axis. Nat Genet 50, 1666-1673, doi:10.1038/s41588-018-0260-3 (2018).

53 Song, Z., Zhang, X., Jia, S., Yelick, P. C. \& Zhao, C. Zebrafish as a Model for Human Ciliopathies. J Genet Genomics 43, 107-120, doi:10.1016/j.jgg.2016.02.001 (2016).

54 Lowery, L. A., De Rienzo, G., Gutzman, J. H. \& Sive, H. Characterization and classification of zebrafish brain morphology mutants. Anat Rec (Hoboken) 292, 94-106, doi:10.1002/ar.20768 (2009).

55 Lowery, L. A. \& Sive, H. Initial formation of zebrafish brain ventricles occurs independently of circulation and requires the nagie oko and snakehead/atp1a1a.1 gene products. Development 132, 2057-2067, doi:10.1242/dev.01791 (2005).

56 Olstad, E. W. et al. Ciliary Beating Compartmentalizes Cerebrospinal Fluid Flow in the Brain and Regulates Ventricular Development. Curr Biol 29, 229-241 e226, doi:10.1016/j.cub.2018.11.059 (2019).

57 Sawamoto, K. New Neurons Follow the Flow of Cerebrospinal Fluid in the Adult Brain. Science 311, 629-632, doi:10.1126/science.1119133 (2006).

58 Demars, M., Hu, Y. S., Gadadhar, A. \& Lazarov, O. Impaired neurogenesis is an early event in the etiology of familial Alzheimer's disease in transgenic mice. J Neurosci Res 88, 2103-2117, doi:10.1002/jnr.22387 (2010).

59 Ma, Q. H., Bagnard, D., Xiao, Z. C. \& Dawe, G. S. A TAG on to the neurogenic functions of APP. Cell Adh Migr 2, 2-8, doi:10.4161/cam.2.1.5790 (2008).

60 Giacomini, A. et al. Inhibition of APP gamma-secretase restores Sonic Hedgehog signaling and neurogenesis in the Ts65Dn mouse model of Down syndrome. Neurobiol Dis 82, 385-396, doi:10.1016/j.nbd.2015.08.001 (2015).

61 Clement, A., Solnica-Krezel, L. \& Gould, K. L. The Cdc14B phosphatase contributes to ciliogenesis in zebrafish. Development 138, 291-302, doi:10.1242/dev.055038 (2011).

62 Fame, R. M. \& Lehtinen, M. K. Emergence and Developmental Roles of the Cerebrospinal Fluid System. Dev Cell 52, 261-275, doi:10.1016/j.devcel.2020.01.027 (2020).

63 Zappaterra, M. W. \& Lehtinen, M. K. The cerebrospinal fluid: regulator of neurogenesis, behavior, and beyond. Cell Mol Life Sci 69, 2863-2878, doi:10.1007/s00018-012-0957-x (2012).

64 LeMay, M. \& Alvarez, N. The relationship between enlargement of the temporal horns of the lateral ventricles and dementia in aging patients with Down syndrome. Neuroradiology 32, 104-107, doi:10.1007/bf00588558 (1990). 
65 Ezratty, E. J., Pasolli, H. A. \& Fuchs, E. A Presenilin-2-ARF4 trafficking axis modulates

880

881

882

883

884

885

886

887

888

889

890

891

892

893

894

895

896

897

898

899

900

901

902

903

904

905

906

907

908

909

910

911

912

913

914

915

916

917

918

919

920

921

922

923

924

925

926

927

Notch signaling during epidermal differentiation. $J$ Cell Biol 214, 89-101, doi:10.1083/jcb.201508082 (2016).

66 Nager, A. R. et al. An Actin Network Dispatches Ciliary GPCRs into Extracellular Vesicles to Modulate Signaling. Cell 168, 252-263 e214, doi:10.1016/j.cell.2016.11.036 (2017).

67 Perez-Gonzalez, R., Gauthier, S. A., Kumar, A. \& Levy, E. The exosome secretory pathway transports amyloid precursor protein carboxyl-terminal fragments from the cell into the brain extracellular space. $J$ Biol Chem 287, 43108-43115, doi:10.1074/jbc.M112.404467 (2012).

68 Spitzer, P. et al. Microvesicles from cerebrospinal fluid of patients with Alzheimer's disease display reduced concentrations of tau and APP protein. Sci Rep 9, 7089, doi:10.1038/s41598-019-43607-7 (2019).

69 Leinonen, V., Kuulasmaa, T. \& Hiltunen, M. iNPH-the mystery resolving. EMBO Mol Med 13, e13720, doi:10.15252/emmm.202013720 (2021).

70 Jeppsson, A., Zetterberg, H., Blennow, K. \& Wikkelsø, C. Idiopathic normal-pressure hydrocephalus: pathophysiology and diagnosis by CSF biomarkers. Neurology 80, 1385-1392, doi:10.1212/WNL.0b013e31828c2fda (2013).

71 Jeppsson, A. et al. Amyloid mis-metabolism in idiopathic normal pressure hydrocephalus. Fluids Barriers CNS 13, 13, doi:10.1186/s12987-016-0037-y (2016).

72 Pyykko, O. T. et al. Cerebrospinal fluid biomarker and brain biopsy findings in idiopathic normal pressure hydrocephalus. PLoS One 9, e91974, doi:10.1371/journal.pone.0091974 (2014).

73 Kim, J. Y. et al. Distinct amyloid precursor protein processing machineries of the olfactory system. Biochem Biophys Res Commun 495, 533-538, doi:10.1016/j.bbrc.2017.10.153 (2018).

74 Reiten, I. et al. Motile-Cilia-Mediated Flow Improves Sensitivity and Temporal Resolution of Olfactory Computations. Curr Biol 27, 166-174, doi:10.1016/j.cub.2016.11.036 (2017).

75 Miyasaka, N. et al. Functional development of the olfactory system in zebrafish. Mech Dev 130, 336-346, doi:10.1016/j.mod.2012.09.001 (2013).

76 Miyasaka, N. et al. Robo2 is required for establishment of a precise glomerular map in the zebrafish olfactory system. Development 132, 1283-1293, doi:10.1242/dev.01698 (2005).

77 Doty, R. L. The olfactory system and its disorders. Semin Neurol 29, 74-81, doi:10.1055/s-0028-1124025 (2009).

78 Trudeau, S. et al. Diagnosis and patterns of hearing loss in children with severe developmental delay. Am J Otolaryngol 42, 102923, doi:10.1016/j.amjoto.2021.102923 (2021).

79 Liu, Y. et al. Hearing loss is an early biomarker in APP/PS1 Alzheimer's disease mice. Neurosci Lett 717, 134705, doi:10.1016/j.neulet.2019.134705 (2020).

80 Omata, Y. et al. Expression of amyloid- $\beta$ in mouse cochlear hair cells causes an earlyonset auditory defect in high-frequency sound perception. Aging 8, 427-439, doi:10.18632/aging.100899 (2016).

81 Kilkenny, C., Browne, W. J., Cuthill, I. C., Emerson, M. \& Altman, D. G. Improving bioscience research reporting: the ARRIVE guidelines for reporting animal research. PLoS Biol 8, e1000412, doi:10.1371/journal.pbio.1000412 (2010).

82 Westerfield, M. The Zebrafish Book : A Guide for the Laboratory Use of Zebrafish. http://zfin.org/zf_info/zfbook/zfbk.html (2000). 
83 Varshney, G. K. et al. A high-throughput functional genomics workflow based on CRISPR/Cas9-mediated targeted mutagenesis in zebrafish. Nature protocols 11, 23572375, doi:10.1038/nprot.2016.141 (2016).

931

932

933

934

935

936

937

938

939

940

941

942

943

944

945

946

947

948

949

84 UniProt, C. UniProt: the universal protein knowledgebase in 2021. Nucleic Acids Res 49, D480-D489, doi:10.1093/nar/gkaa1100 (2021).

85 Banote, R. K. et al. Amyloid precursor protein-b facilitates cell adhesion during early development in zebrafish. Sci. Rep. 10, 10127, doi:10.1038/s41598-020-66584-8 (2020).

86 Kimmel, C. B., Ballard, W. W., Kimmel, S. R., Ullmann, B. \& Schilling, T. F. Stages of embryonic development of the zebrafish. Dev Dyn 203, 253-310, doi:10.1002/aja.1002030302 (1995).

87 Lauter, G., Söll, I. \& Hauptmann, G. Sensitive whole-mount fluorescent in situ hybridization in zebrafish using enhanced tyramide signal amplification. Methods Mol. Biol. 1082, 175-185, doi:10.1007/978-1-62703-655-9_12 (2014).

88 Lashley, T. et al. A comparative clinical, pathological, biochemical and genetic study of fused in sarcoma proteinopathies. Brain 134, 2548-2564, doi:10.1093/brain/awr160 (2011).

89 Livak, K. J. \& Schmittgen, T. D. Analysis of relative gene expression data using realtime quantitative PCR and the 2(-Delta Delta C(T)) Method. Methods (San Diego, Calif.) 25, 402-408, doi:10.1006/meth.2001.1262 (2001).

90 Gutzman, J. H. \& Sive, H. Zebrafish brain ventricle injection. $J$ Vis Exp, doi:10.3791/1218 (2009).

950

951

\section{Acknowledgements}

952

We thank Elisa Alexandersson and Katarina Türner Stenström for fish maintenance and the

Centre for Cellular Imaging at the University of Gothenburg and the National Microscopy

954 Infrastructure (VR-RFI 2016-00968) for microscopy support. We also thank Debora Kaminski

955 for the mouse brains samples, Nathalie Jurish-Yaksi (Norwegian University of Science and

956 Technology - NTNU) and Jean-François Papon (Public Hospital Network of Paris (AP-HP))

957 for insight and thoughtful discussions about cilia. Images were drawn by Jasmine Chebli.

958

\section{Author contributions}

960 JC: Conceptualization, Formal analysis, Investigation, Visualization, Methodology, Data

961 curation, Project administration, Writing - original draft, Writing - review and editing. MR,

962 TL, AO and BE: Formal analysis, Writing - review and editing. HZ: Resources, Supervision, 
963 Funding acquisition, Writing - original draft, Project administration, Writing - review and 964 editing. AA: Conceptualization, Supervision, Formal analysis, Investigation, Visualization, 965 Methodology, Writing - original draft, Project administration, Writing - review and editing. All 966 authors reviewed and approved the final manuscript.

967

968 Author ORCIDs: JC: 0000-0003-0791-3198, TL: 0000-0001-7389-0348, AO: 0000-0002969 5758-7397, HZ: 0000-0003-3930-4354, AA: 0000-0002-4715-9225.

970

\section{$971 \quad$ Additional information}

972 Competing interests: The authors have no competing interests of relevance to the current 973 manuscript.

974 Funding: The study was supported by grants from the Swedish Research Council (\#2018975 02532), the European Research Council (\#681712), Stiftelsen för Gamla Tjänarinnor, and 976 Hjärnfonden, Sweden. HZ is a Wallenberg Scholar. TL is funded by an Alzheimer's Research 977 UK senior fellowship. The Queen Square Brain Bank for Neurological Disorders is supported 978 by the Reta Lila Weston Institute for Neurological Studies. 
981 Figure 1. Expression pattern of appa and appb mRNA. (A,B) Schematic representations of 982 head and ventricle morphology of a 30 hpf zebrafish larvae, lateral (A) and dorsal (B) view. $983(\mathbf{C , H})$ Whole-mount fluorescent in situ of appa $(\mathbf{C})$ and $a p p b(\mathbf{H})$ in 30 hpf WT zebrafish larvae. 984 Single focal planes, dorsal to ventral, of whole-mount larvae of appa (D-G) and appb (I-L) 985 probe. (M) Schematic view of focal plane of the dorsal area of the brain ventricle. (N-Q) Single 986 focal plane at high magnification $(40 \mathrm{x})$ of $\operatorname{appa}(\mathbf{N}, \mathbf{O})$ and $\operatorname{appb}(\mathbf{P}, \mathbf{Q})$ probes. $\mathrm{T}=$ telencephalic 987 ventricle, $\mathrm{D} / \mathrm{M}=$ diencephalic/mesencephalic ventricle, $\mathrm{R}=$ rhombencephalic ventricle, $\mathrm{Ob}=$ 988 olfactory bulb, $\mathrm{Oe}=$ olfactory epithelium, $\mathrm{P}=$ pituitary gland, $\mathrm{Le}=$ lens, $\mathrm{Ot}=$ optic tectum, $\mathrm{Tg}=$ trigeminal ganglia, $\mathrm{Rh}=$ rhombomeres, $\mathrm{Ov}=$ otic vesicle. Magnification: $(\mathbf{C}-\mathbf{L})=20 \mathrm{x},(\mathbf{N}-\mathbf{Q})=$ 40x. Scale bar: $(\mathbf{C})=100 \mu \mathrm{m},(\mathbf{D})=50 \mu \mathrm{m},(\mathbf{N})=25 \mu \mathrm{m}$. * indicates ventricular space and arrows 991 highlight expression.

Figure 2. Localization of App protein to cilia of the olfactory sensory neurons and otic

994

995

996

997

998

999

1000

1001

1002

1003

1004 vesicle in 31 hpf larvae. Cilia as shown by immunostaining for acetylated tubulin (magenta) and App (green) of the olfactory sensory neurons in the nose epithelium $(\mathbf{A})$ and the otic vesicle (B-C). In (A), dotted lines demarcate the cilia from the nasal cavity (see asterisk). (A') App (green) is found along the cilia and accumulating at their base. Otic vesicle of $24 \mathrm{hpf}(\mathbf{B})$ and 31 hpf larvae (C). In (B), glutamylated tubulin (cyan) highlights the base of the cilia outlined by acetylated tubulin staining (magenta). (B) Overview of the kinocilia and stereocilia of the otic vesicle. The white asterisks indicate accumulation of App (green) at the base of the cilia bundles. (B') Magnification of cilia outlined in (B). (B'") Increased intensity of the green channel to detect App (arrows) in kinocilia. Otic vesicle in 31hpf zebrafish larvae (C) with close-up (C') showing App puncta (green) along the kinocilia. Intensity profiles of acetylated tubulin (magenta) and App (green) staining from the kinocilia (D-F). In (D), the intensity profile 
1005 of the whole length of the kinocilium is plotted whereas profiles of cross-section lines are 1006 plotted with a visible App puncta $(\mathbf{E})$ and without $(\mathbf{F})$. The dotted lines $\left(\mathbf{C}^{\prime}\right)$ indicate the 1007 kinocilium and cross-sections. Magnification: $(\mathbf{A}-\mathbf{C})=40 x$. Scale bar: $(\mathbf{A})=5 \mu \mathrm{m},(\mathbf{B})=10 \mu \mathrm{m}$, $1008 \quad\left(\mathbf{B}^{\prime}\right)=4 \mu \mathrm{m},\left(\mathbf{B}^{\prime \prime}\right)=2 \mu \mathrm{m},(\mathbf{C})=10 \mu \mathrm{m}$.

1009

1010 Figure 3. App localizes to the cilia decorating the ventricle of larvae and ependymal cells in

1011 adult zebrafish. (A) Schematic representations of head and ventricle morphology in 30 hpf 1012 zebrafish larvae, dorsal view. (B) Dorsal view of ventricle immunostained for App (green) and 1013 acetylated tubulin (magenta) in $30 \mathrm{hpf}$ WT zebrafish larvae. (C) Close-up of cilia (magenta) 1014 and App (green). (D) Schematic outline of adult zebrafish brain, lateral view. (E-F) Coronal 1015 section of adult zebrafish brain and the central canal (see asterisk). Cell nuclei labeled with 1016 DAPI (blue), acetylated tubulin (magenta), App (green). (F) Ependymal motile cilia (magenta) 1017 of the central canal with App (green) accumulation along cilia. Magnification: $(\mathbf{B}, \mathbf{E})=10 \mathrm{x},(\mathbf{C}$, $1018 \quad$ F $)=60 x$. Scale bar: $(\mathbf{B})=50 \mu \mathrm{m},(\mathbf{C})=1 \mu \mathrm{m},(\mathbf{E})=500 \mu \mathrm{m},(\mathbf{F})=10 \mu \mathrm{m}$.

Figure 4. APP is localized to the ependymal cilia in adult mouse. (A) Schematic representation 1021 of adult mouse brain ventricular system, sagittal view. (B) Overview of sagittal section from 1022 adult mouse brain and the third ventricle (see dotted square in (A)) for cell nuclei stained with DAPI (blue), acetylated tubulin (magenta), APP (green). (C-D) Close-up of ependymal cells and their cilia tufts (magenta) and APP expression with anti-APP Y188 antibody $(\mathbf{C})$ and 22C11 antibody (D). $\mathrm{LV}=$ lateral ventricle, $\mathrm{III}=$ third ventricle, $\mathrm{IV}=$ fourth ventricle, $\mathrm{H}=$ hippocampus,

$1026 \mathrm{Cp}=$ choroid plexus, $\mathrm{Ep}=$ ependyme. Magnification: $(\mathbf{B})=10 \mathrm{x},(\mathbf{C}, \mathbf{D})=60 \mathrm{x}$. Scale bar: $(\mathbf{B})=$ $1027200 \mu \mathrm{m},(\mathbf{C}, \mathbf{D})=10 \mu \mathrm{m}$. 
1029 Figure 5. APP is localized to human ependymal cilia. (A) Brightfield overview of a human

1030 brain section of the caudate nucleus immunostained with an anti-acetylated tubulin antibody

1031 reveals the different cellular layers (I-IV): (I) ependyme layer with motile cilia orienting

1032 towards the ventricle lumen, (II) cellular extensions connecting the ependymal cells, (III)

1033 cellular layer dense in astrocytes, (IV) brain parenchyma. (B-E) Higher magnifications of the

1034 ependymal layer show clear cilia (acetylated tubulin (B,D)) and APP (Y188 antibody (C,E))

1035 accumulation within ependymal cells and along ependymal cilia. (E'). Arrows highlight 1036 ependymal cilia tufts in the ventricular lumen. White asterisks indicate broken or absent cilia.

1037 Dotted lines delimitate the ependymal cell layer. Magnification: $(\mathbf{A})=20 x,(\mathbf{B}-\mathbf{C})=40 \mathrm{x},(\mathbf{D}-\mathbf{E})=$ 1038 100x. Scale bar: $(\mathbf{A})=5 \mu \mathrm{m},(\mathbf{B})=10 \mu \mathrm{m},(\mathbf{D})=2 \mu \mathrm{m}$.

1039

1040 Figure 6. Generation of $a p p a^{-/}$and analysis of $a p p a^{-/} a p p b^{-/}$double mutant zebrafish. (A)

1041 Schematic outline of the appa gene with exons (black box) and UTR regions (white box).

1042 sgRNA used to target exon 2 with protospacer adjacent motif (PAM) in red and the sgRNA

1043 target sequence underlined. (B) Sanger sequencing chromatogram of exon 2 in wild-type and 1044 appa $^{-/}$zebrafish. (C) Schematic drawing of the wild-type Appa protein (738 aa) with epitopes 1045 of antibodies (dotted squares) used above and the hypothetical truncated Appa (109 aa) protein 1046 produced in appa mutant below. (D) qPCR quantification of appa and appb mRNA levels in 1047 wild-type and appa $^{-/} a p p b^{-/-}$mutants at 24 hpf. (E) Western blot of 3 dpf whole larvae zebrafish 1048 with antibodies against 22C11 and App (Y188). Alpha-tubulin is used as loading control. Blots 1049 cropped from the same original gel and grouped. Quantification of band intensity are shown 1050 relative to control. Data are reported as mean \pm SD. $* * \rho<0.05$, **** $\rho<0.001$. qPCR $\mathrm{n}=5$, 1051 WB $\mathrm{n}=3 . \mathrm{SP}=$ signal peptide, $\mathrm{E} 1=$ extracellular domain, $\mathrm{ED}=$ extension domain, $\mathrm{AcD}=$ acidic 1052 domain, $\mathrm{E} 2=$ extracellular domain $2, \mathrm{JMR}=$ juxtamembrane region, $\mathrm{A} \beta=$ amyloid beta, $\mathrm{TM}=$ 1053 transmembrane, $\mathrm{AICD}=$ amyloid intracellular domain. 
1054 Figure 7. Longer cilia of dorsal brain ventricle neuroepithelium in $a p p a^{-/} a p p b^{-/}$larvae 1055 zebrafish. At 30hpf, appa ${ }^{-/} a p p b^{-/}$exhibit longer diencephalic/mesencephalic ventricle cilia 1056 than WT. Data are reported as mean \pm SEM. $* * * * \rho<0.001 . \mathrm{n}=10 \mathrm{WT}$ (1091 cilia), 16 appa $^{-/-}$ $1057 a p p b^{-/-}(1511$ cilia).

Figure 8. Structural integrity of ependymal cilia in WT and $a p p a^{-/} a p p b^{-/}$zebrafish.

1060 Transmission electron microscopy of adult zebrafish ependymal cilia of WT (A-D) and appa $^{-/}$

$1061 a p p b^{-/}$mutant $(\mathbf{E}-\mathbf{H})$ adult zebrafish. (A,E) Overview of ependymal cilia of the central canal.

1062 (B,F) Longitudinal view on the axoneme of the cilia composing its core, the transition zone including the ciliary pit between the cilia core and the cellular membrane and the basal body

1064 containing the cilia centrioles, highlighted with increased signal. In $(\mathbf{C}, \mathbf{G})$, cross-sections of cilia. (D-H) Zoom on cross-section of individual cilia showing $(9+2)$ microtubule doublet organization. Scale bar: $(\mathbf{A}, \mathbf{E})=1 \mu \mathrm{m},(\mathbf{B}-\mathbf{C}, \mathbf{F}-\mathbf{G})=200 \mathrm{~nm},(\mathbf{D}, \mathbf{H})=50 \mathrm{~nm}$.

Figure 9. The $a p p a^{-/} a p p b^{-/} 2$ dpf larvae zebrafish exhibit smaller brain ventricle. Dorsal 3D 1069 surface rending of confocal stacks taken from brain ventricles of dextran injected 2 dpf zebrafish larvae (A). Quantification of total ventricle surface area and volume show that both

1071 are decreased in $a p p a^{-/-} a p p b^{-/-}$larvae $(\mathbf{B})$. Lateral 3D surface rending of confocal stacks from 1072 brain ventricles of dextran injected 2 dpf zebrafish larvae with close up on diencephalic

1073 ventricle (C). Quantification of surface area and volume of the diencephalic ventricle in WT 1074 and $\mathrm{appa}^{-/-} a p p b^{-/-}$larvae (D). Measurement of gross ventricle morphology at $2 \mathrm{dpf}$ WT and $a p p a^{-}$ $1075{ }^{\prime}{ }^{\prime} a p p b^{-/}$larvae as the length $(\mathbf{E})$. Distance between rostral to caudal, diencephalon ventricle 1076 sagittal length, amplitude and height show no significant difference in mutants (F). Data are 
1077 reported as mean \pm SEM. $* * \rho<0.01$, **** $\rho<0.001 . \mathrm{n}:(\mathbf{B}) \mathrm{WT}=19$, appa $^{-/}$appb $^{-/}=34$, (D)

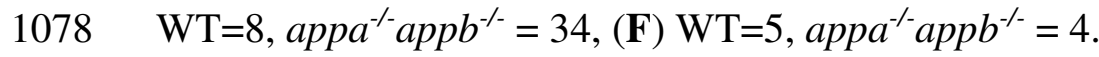

1079 


\section{Supplementary Files}

This is a list of supplementary files associated with this preprint. Click to download.

- SupplementarymaterialsCheblietal.pdf 